\title{
Path-integral simulations with fermionic and bosonic reservoirs: Transport and dissipation in molecular electronic junctions
}

\author{
Lena Simine and Dvira Segal \\ Chemical Physics Theory Group, Department of Chemistry, \\ University of Toronto, 80 Saint George St. Toronto, Ontario, Canada M5S $3 H 6$
}

(Dated: June 1, 2022)

\begin{abstract}
We expand iterative numerically-exact influence functional path-integral tools and present a method capable of following the nonequilibrium time evolution of subsystems coupled to multiple bosonic and fermionic reservoirs simultaneously. Using this method, we study the real-time dynamics of charge transfer and vibrational mode excitation in an electron conducting molecular junction. We focus on nonequilibrium vibrational effects, particularly, the development of vibrational instability in a current-rectifying junction. Our simulations are performed by assuming large molecular vibrational anharmonicity (or low temperature). This allows us to truncate the molecular vibrational mode to include only a two-state system. Exact numerical results are compared to perturbative Master equation calculations demonstrating an excellent agreement in the weak electron-phonon coupling regime. Significant deviations take place only at strong coupling. Our simulations allow us to quantify the contribution of different transport mechanisms, coherent dynamics and inelastic transport, in the overall charge current. This is done by studying two model variants: The first admits inelastic electron transmission only, while the second one allows for both coherent and incoherent pathways.
\end{abstract}




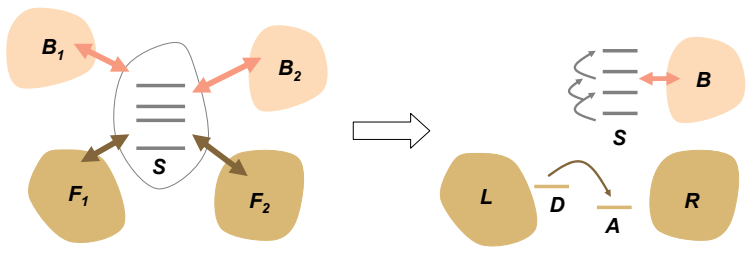

FIG. 1. Left Panel: Generic setup considered in this work, including a subsystem $(S)$ coupled to multiple fermionic $(F)$ and bosonic $(B)$ reservoirs. Right panel: Molecular electronic realization with two metals, $L$ and $R$, connected by two electronic levels, $D$ and $A$. Electronic transitions in this junction are coupled to excitation/de-excitation processes of a particular, anharmonic, vibrational mode that plays the role of the "subsystem". This mode may dissipate its excess energy to a secondary phonon bath $B$.

\section{INTRODUCTION}

Following the quantum dynamics of an open-dissipative many-body system with multiple bosonic and fermionic reservoirs in a nonequilibrium state, beyond the linear response regime, is a significant theoretical and computational challenge. In the realm of molecular conducting junctions, we should describe the out-of-equilibrium dynamics of the molecular unit while handling both electrons and molecular vibrations, accounting for many-body effects such as electron-electron, phonon-phonon and electron-phonon interactions. Given this complexity, studies in this field are mostly focused on steady-state properties, using e.g., scattering theory [1 3 ], while ignoring vibrational nonequilibrium effects. Perturbative treatments (in either the molecule-leads coupling parameter or the electron-phonon interaction energy) are commonly used, including the nonequilibrium Green's function technique [4] 8] and Master equation approaches [5, 9-13]. For following the real-time dynamics of such systems, involved methods have been recently developed, e.g., semiclassical approaches [14, 15$]$.

In this work, we extend numerically-exact path-integral methods, and follow the dynamics of a subsystem coupled to multiple out-of-equilibrium bosonic and fermionic reservoirs. The technique is then applied on a molecular junction realization, with the motivation to address basic problems in the field of molecular electronics. Particularly, in this work we consider the 
dynamics and steady-state properties of a conducting molecular junction acting as a charge rectifier. A scheme of the generic setup and a particular molecular junction realization are depicted in Fig. [1.

The time evolution scheme developed in this paper treats both bosonic and fermionic reservoirs. This is achieved by combining two related iterative path-integral methods: (i) The quasi-adiabatic path-integral approach (QUAPI) of Makri et al. [16], applicable for the study of subsystem-boson models, and (i) the recently developed influence-functional pathintegral (INFPI) technique [17], able to produce the dynamics of subsystems in contact with multiple fermi baths. The latter method (INFPI) essentially generalizes QUAPI. It relies on the observation that in out-of-equilibrium (and/or finite temperature) situations bath correlations have a finite range, allowing for their truncation beyond a memory time dictated by the voltage-bias and the temperature. Taking advantage of this fact, an iterativedeterministic time-evolution scheme can be developed, where convergence with respect to the memory length can in principle be reached.

The principles of the INFPI approach have been detailed in [17], where it has been adopted for investigating dissipation effects in the nonequilibrium spin-fermion model and charge occupation dynamics in correlated quantum dots. Recently, it was further utilized for examining the effect of a magnetic flux on the intrinsic coherence dynamics in a double quantum dot system [18], and for studying relaxation and equilibration dynamics in finite metal grains [19].

Numerically-exact methodologies are typically limited to simple models; analytic results are further restricted to specific parameters. The Anderson-Holstein (AH) model has been studied extensively in this context. In this model the electronic structure of the molecule is represented by a single spinless electronic level, with electron occupation on the dot coupled to the displacement of a single oscillator mode, representing an internal vibration. This vibration may connect with a secondary phonon bath, representing a larger phononic environment (internal modes, solvent). The AH model has been simulated exactly with the secondary phonon bath, using a a real-time path-integral Monte Carlo approach [20], and by extending the multilayer multiconfiguration time-dependent Hartree method to include fermionic degrees of freedom [21]. More recently, the model has been simulated by adopting the iterative-summation of path-integral approach [22 24].

In this paper, we examine a variant of the AH model, the Donor (D)-Acceptor (A) elec- 
tronic rectifier model [25]. This model incorporates nonlocal electron-vibration interactions: electronic transitions between the two molecular states, A and D, are coupled to a particular internal molecular vibrational mode. Within this simple system, we are concerned with the development of vibrational instability: Significant molecular heating can take place once the D level is lifted above the A level, as the excess electronic energy is used to excite the vibrational mode. This process may ultimately lead to junction instability and breakdown [26]. We have recently studied a variant of this model (excluding direct D-A tunneling element), using a Master equation method, by working in the weak electron-phonon coupling limit. [27]. An important observation in that work has been that since the development of this type of instability is directly linked to the breakdown of the detailed balance relation above a certain bias (resulting in an enhanced vibrational excitation rate constant, over relaxation), it suffices to describe the vibrational mode as a truncated two-level system. In this picture, population inversion in the two-state system evinces on the development of vibrational instability.

Our objectives here are threefold: (i) To present a numerically-exact iterative scheme for following the dynamics of a quantum system driven to a nonequilibrium steady-state due to its coupling to multiple bosonic and fermionic reservoirs. (ii) To demonstrate the applicability of the method in the field of molecular electronics. Particularly, to explore the development of vibrational instability in conducting molecules. (iii) To evaluate the performance and accuracy of standard-perturbative Master equation treatments, by comparing their predictions to exact results. Since Master equation techniques are extensively used for explaining charge transfer phenomenology, scrutinizing their validity and accuracy is an important task.

The plan of the paper is as follows. In Sec. II] we introduce the path-integral formalism. We describe the iterative time evolution scheme in Sec. III, by exemplifying it to the case of a spin subsystem. Sec. IV describes a molecular electronics application, and we follow both electrons and vibrational dynamics in a dissipative molecular rectifier. Sec. $\nabla$ concludes. For simplicity, we use the conventions $\hbar \equiv 1$, electron charge $e \equiv 1$, and Boltzmann constant $k_{B}=1$. 


\section{PATH-INTEGRAL FORMULATION}

We consider a multi-level subsystem, with the Hamiltonian $H_{S}$, coupled to multiple bosonic $(B)$ and fermionic $(F)$ reservoirs that are prepared in an out-of-equilibrium initial state. The total Hamiltonian $H$ is written as

$$
H=H_{S}+H_{B}+H_{F}+V_{S B}+V_{S F} .
$$

In the energy representation of the isolated subsystem, its Hamiltonian can be written as

$$
H_{S}=\sum_{s} \epsilon_{s}|s\rangle\left\langle s\left|+\sum_{s \neq s^{\prime}} v_{s, s^{\prime}}\right| s\right\rangle\left\langle s^{\prime}\right| .
$$

The Hamiltonian $H_{F}$ may comprise of multiple fermionic baths, and similarly, $H_{B}$ may contain more than a single bosonic reservoir. The terms $V_{S F}$ and $V_{S B}$ include the coupling of the subsystem to the fermionic and bosonic environments, respectively. Coupling terms which directly link the subsystem to both bosonic and fermionic degrees of freedom are not included. However, $V_{S B}$ and $V_{S F}$ may contain non-additive contributions with their own set of reservoirs. For example, $V_{S F}$ may admit subsystem assisted tunneling terms, between separate fermionic baths (metals), see Fig. 1.

We are interested in the time evolution of the reduced density matrix $\rho_{S}(t)$. This quantity is obtained by tracing the total density matrix $\rho$ over the bosonic and fermionic reservoirs' degrees of freedom

$$
\rho_{S}(t)=\operatorname{Tr}_{B} \operatorname{Tr}_{F}\left[e^{-i H t} \rho(0) e^{i H t}\right]
$$

We also study the dynamics of certain expectation values, for example, charge current and energy current. The time evolution of an operator $A$ can be calculated using the relation

$$
\begin{aligned}
\langle A(t)\rangle & =\operatorname{Tr}[\rho(0) A(t)] \\
& =\lim _{\lambda \rightarrow 0} \frac{\partial}{\partial \lambda} \operatorname{Tr}\left[\rho(0) e^{i H t} e^{\lambda A} e^{-i H t}\right] .
\end{aligned}
$$

Here $\lambda$ is a real number, taken to vanish at the end of the calculation. When unspecified, the trace is performed over the subsystem states and all the environmental degrees of freedom. In what follows, we detail the path-integral approach for the calculation of the reduced density matrix. Section ПIE presents expressions useful for time-evolving expectation values of operators. 
As in standard path-integral approaches, we decompose the time evolution operator into a product of $N$ exponentials, $e^{i H t}=\left(e^{i H \delta t}\right)^{N}$ where $t=N \delta t$, and define the discrete time evolution operator $\mathcal{G} \equiv e^{i H \delta t}$. Using the Trotter decomposition, we approximate $\mathcal{G}$ by

$$
\mathcal{G} \sim \mathcal{G}_{\mathcal{F}} \mathcal{G}_{\mathcal{B}} \mathcal{G}_{\mathcal{S}} \mathcal{G}_{\mathcal{B}} \mathcal{G}_{\mathcal{F}}
$$

where we define

$$
\begin{aligned}
\mathcal{G}_{\mathcal{F}} & \equiv e^{i\left(H_{F}+V_{S F}\right) \delta t / 2}, \quad \mathcal{G}_{\mathcal{B}} \equiv e^{i\left(H_{B}+V_{S B}\right) \delta t / 2} \\
\mathcal{G}_{\mathcal{S}} & \equiv e^{i H_{S} \delta t}
\end{aligned}
$$

Note that the breakup of the subsystem-bath term, $e^{i\left(H_{F}+V_{S F}+H_{B}+H_{S B}\right) \delta t / 2} \sim \mathcal{G}_{\mathcal{B}} \mathcal{G}_{\mathcal{F}}$, is exact if the commutator $\left[V_{S B}, V_{S F}\right]$ vanishes. This fact allows for an exact separation between the bosonic and fermionic influence functionals, as we explain below. This commutator nullifies if the fermionic and bosonic baths couple to commuting subsystem degrees of freedom, for example, $V_{S B} \propto|s\rangle\langle s|$ and $V_{S F} \propto\left|s^{\prime}\right\rangle\left\langle s^{\prime}\right|$.

As an initial condition, we assume that at time $t=0$ the subsystem and the baths are decoupled, $\rho(0)=\rho_{S}(0) \otimes \rho_{B} \otimes \rho_{F}$, and the baths are prepared in a nonequilibrium (biased) state. For example, we may include in $H_{F}$ two Fermi seas that are prepared each in a grand canonical state with different chemical potentials and temperatures. The overall time evolution can be represented by a path-integral over the subsystem states,

$$
\begin{aligned}
& \left\langle s_{N}^{+}\left|\rho_{S}(t)\right| s_{N}^{-}\right\rangle \\
& =\sum_{s_{0}^{ \pm}} \sum_{s_{1}^{ \pm}} \ldots \sum_{s_{N-1}^{ \pm}} \operatorname{Tr}_{B} \operatorname{Tr}_{F}\left[\left\langle s_{N}^{+}\left|\mathcal{G}^{\dagger}\right| s_{N-1}^{+}\right\rangle\left\langle s_{N-1}^{+}\left|\mathcal{G}^{\dagger}\right| s_{N-2}^{+}\right\rangle \ldots\left\langle s_{0}^{+}|\rho(0)| s_{0}^{-}\right\rangle \ldots\left\langle s_{N-2}^{-}|\mathcal{G}| s_{N-1}^{-}\right\rangle\left\langle s_{N-1}^{-}|\mathcal{G}| s_{N}^{-}\right\rangle\right] .
\end{aligned}
$$

Here $s_{k}^{ \pm}$represents the discrete path on the forward $(+)$and backward $(-)$contour. The calculation of each discrete term is done by introducing four additional summations, e.g.,

$$
\left\langle s_{k}^{-}|\mathcal{G}| s_{k+1}^{-}\right\rangle=\sum_{f_{k}^{-}} \sum_{g_{k}^{-}} \sum_{m_{k}^{-}} \sum_{n_{k}^{-}}\left\langle s_{k}^{-}\left|\mathcal{G}_{\mathcal{F}}\right| f_{k}^{-}\right\rangle\left\langle f_{k}^{-}\left|\mathcal{G}_{\mathcal{B}}\right| m_{k}^{-}\right\rangle\left\langle m_{k}^{-}\left|\mathcal{G}_{\mathcal{S}}\right| n_{k}^{-}\right\rangle\left\langle n_{k}^{-}\left|\mathcal{G}_{\mathcal{B}}\right| g_{k}^{-}\right\rangle\left\langle g_{k}^{-}\left|\mathcal{G}_{\mathcal{F}}\right| s_{k+1}^{-}\right\rangle \text {. }
$$

We substitute Eq. (8) into Eq. (7), further utilizing the factorized subsystem-reservoirs initial condition as mentioned above, and find that the function under the sum can be 
written as a product of separate terms,

$$
\left\langle s_{N}^{+}\left|\rho_{S}(t)\right| s_{N}^{-}\right\rangle=\sum_{\mathbf{s}^{ \pm}} \sum_{\mathbf{f}^{ \pm}} \sum_{\mathbf{g}^{ \pm}} \sum_{\mathbf{m}^{ \pm}} \sum_{\mathbf{n}^{ \pm}} I_{S}\left(\mathbf{m}^{ \pm}, \mathbf{n}^{ \pm}, s_{0}^{ \pm}\right) I_{F}\left(\mathbf{s}^{\prime \pm}, \mathbf{f}^{ \pm}, \mathbf{g}^{ \pm}\right) I_{B}\left(\mathbf{f}^{ \pm}, \mathbf{m}^{ \pm}, \mathbf{n}^{ \pm}, \mathbf{g}^{ \pm}\right) .
$$

Here $I_{S}$ follows the subsystem $\left(H_{S}\right)$ free evolution. The term $I_{F}$ is referred to as a fermionic "influence functional" (IF), and it contains the effect of the fermionic degrees of freedom on the subsystem dynamics. Similarly, $I_{B}$, the bosonic IF, describes how the bosonic degrees of freedom affect the subsystem. Bold letters correspond to a path, for example, $\mathbf{m}^{ \pm}=$ $\left\{m_{0}^{ \pm}, m_{1}^{ \pm}, \ldots, m_{N-1}^{ \pm}\right\}$. We also define the path $\mathbf{s}^{ \pm}=\left\{s_{0}^{ \pm}, s_{1}^{ \pm}, \ldots, s_{N-1}^{ \pm}\right\}$, and the associate

path which covers $N+1$ points, $\mathbf{s}^{ \pm}=\left\{s_{0}^{ \pm}, s_{1}^{ \pm}, \ldots, s_{N-1}^{ \pm}, s_{N}^{ \pm}\right\}$. Given the product structure of Eq. (9), the subsystem, bosonic and the fermionic terms can be independently evaluated, while coordinating their path. Explicitly, the elements in Eq. (9) are given by

$$
\begin{aligned}
I_{S} & =\left\langle s_{0}^{+}\left|\rho_{S}(0)\right| s_{0}^{-}\right\rangle \Pi_{k=0, \ldots, N-1}\left\langle m_{k}^{-}\left|\mathcal{G}_{S}\right| n_{k}^{-}\right\rangle\left\langle n_{k}^{+}\left|\mathcal{G}_{S}^{\dagger}\right| m_{k}^{+}\right\rangle \\
I_{F} & =\operatorname{Tr}_{F}\left[\left\langle s_{N}^{+}\left|\mathcal{G}_{F}^{\dagger}\right| g_{N-1}^{+}\right\rangle\left\langle f_{N-1}^{+}\left|\mathcal{G}_{F}^{\dagger}\right| s_{N-1}^{+}\right\rangle \ldots\right. \\
& \times\left\langle s_{1}^{+}\left|\mathcal{G}_{F}^{\dagger}\right| g_{0}^{+}\right\rangle\left\langle f_{0}^{+}\left|\mathcal{G}_{F}^{\dagger}\right| s_{0}^{+}\right\rangle \rho_{F}\left\langle s_{0}^{-}\left|\mathcal{G}_{F}\right| f_{0}^{-}\right\rangle\left\langle g_{0}^{-}\left|\mathcal{G}_{F}\right| s_{1}^{-}\right\rangle \ldots \\
& \left.\times\left\langle s_{N-1}^{-}\left|\mathcal{G}_{F}\right| f_{N-1}^{-}\right\rangle\left\langle g_{N-1}^{-}\left|\mathcal{G}_{F}\right| s_{N}^{-}\right\rangle\right] \\
I_{B} & =\operatorname{Tr}_{B}\left[\left\langle g_{N-1}^{+}\left|\mathcal{G}_{B}^{\dagger}\right| n_{N-1}^{+}\right\rangle\left\langle m_{N-1}^{+}\left|\mathcal{G}_{B}^{\dagger}\right| f_{N-1}^{+}\right\rangle \ldots\right. \\
& \times\left\langle g_{0}^{+}\left|\mathcal{G}_{B}^{\dagger}\right| n_{0}^{+}\right\rangle\left\langle m_{0}^{+}\left|\mathcal{G}_{B}^{\dagger}\right| f_{0}^{+}\right\rangle \rho_{B}\left\langle f_{0}^{-}\left|\mathcal{G}_{B}\right| m_{0}^{-}\right\rangle\left\langle n_{0}^{-}\left|\mathcal{G}_{B}\right| g_{0}^{-}\right\rangle \ldots \\
& \left.\times\left\langle f_{N-1}^{-}\left|\mathcal{G}_{B}\right| m_{N-1}^{-}\right\rangle\left\langle n_{N-1}^{-}\left|\mathcal{G}_{B}\right| g_{N-1}^{-}\right\rangle\right] .
\end{aligned}
$$

The dynamics in Eq. (9) can be retrieved by following an iterative scheme, by using the principles of the INFPI approach [17]. In the next section we illustrate this evolution with a spin subsystem.

\section{ITERATIVE TIME EVOLUTION SCHEME}

We consider here the spin-boson-fermion model. It includes a two-state subsystem that is coupled through its polarization to bosonic and fermionic reservoirs. With this relatively simple model, we exemplify the iterative propagation technique, see Secs. IIIA-IIE, Relevant expressions for a multi-level subsystem and general interaction form are included in Sec. IIIF, 


\section{A. spin-boson-fermion model}

The spin-fermion model, with a qubit, spin, coupled to a fermionic bath is kindred to the eminent spin-boson model, describing a qubit interacting with bosonic environment. It is also related to the Kondo model [28], only lacking direct coupling of the reservoir degrees of freedom to spin-flip processes. It provides a minimal setting for the study of dissipation and decoherence effects in the presence of nonequilibrium reservoirs [29 32]. Here we put together the spin-boson and the spin-fermion models, and present it in the general form,

$$
\begin{aligned}
H_{S} & =\Delta \sigma_{x}+B \sigma_{z}, \\
H_{F} & =\sum_{j} \epsilon_{j} c_{j}^{\dagger} c_{j}+\sum_{j \neq j^{\prime}} v_{j, j^{\prime}}^{F} c_{j}^{\dagger} c_{j^{\prime}} \\
V_{S F} & =\sigma_{z} \sum_{j, j^{\prime}} \xi_{j, j^{\prime}}^{F} c_{j}^{\dagger} c_{j^{\prime}} . \\
H_{B} & =\sum_{p} \omega_{p} b_{p}^{\dagger} b_{p}+\sum_{p, p^{\prime}} v_{p, p^{\prime}}^{B} b_{p}^{\dagger} b_{p^{\prime}}, \\
V_{S B} & =\sigma_{z} \sum_{p} \xi_{p}^{B}\left(b_{p}^{\dagger}+b_{p}\right)+\sigma_{z} \sum_{p, p^{\prime}} \zeta_{p, p^{\prime}}^{B} b_{p}^{\dagger} b_{p^{\prime}} .
\end{aligned}
$$

The subsystem includes only two states, with an energy gap $2 B$ and a tunneling splitting $2 \Delta$. This minimal subsystem is coupled here through its polarization to a set of boson and fermion degrees of freedom, where $\sigma_{z}$ and $\sigma_{x}$ denote the $z$ and $x$ Pauli matrices for a two-state subsystem, respectively. $b_{p}$ stands for a bosonic operator, to destroy a mode of frequency $\omega_{p}$, similarly, $c_{j}$ is a fermionic operator, to annihilate an electron of energy $\epsilon_{j}$ (we assume later a linear dispersion relation). In this model, spin polarization couples to harmonic displacements, to scattering events between electronic states in the metals (fermi reservoirs), and to scattering evens between different modes in the harmonic bath. Since the commutator between the interaction terms vanish, $\left[V_{S F}, V_{S B}\right]=0$, the separation between the bosonic and fermionic IFs is exact. Moreover, since the fermionic and bosonic operators couple both to $\sigma_{z}$, we immediately note that $f_{k}^{ \pm}=s_{k}^{ \pm}, m_{k}^{ \pm}=f_{k}^{ \pm}, n_{k}^{ \pm}=g_{k}^{ \pm}$and $g_{k}^{ \pm}=s_{k+1}^{ \pm}$. Eq. (9) then simplifies to

$$
\left\langle s_{N}^{+}\left|\rho_{S}(t)\right| s_{N}^{-}\right\rangle=\sum_{\mathbf{s}^{ \pm}} I_{S}\left(\mathbf{s}^{ \pm}\right) I_{F}\left(\mathbf{s}^{\prime \pm}\right) I_{B}\left(\mathbf{s}^{\prime \pm}\right)
$$

where we recall the definitions of the paths $\mathbf{s}^{ \pm}=\left\{s_{0}^{ \pm}, s_{1}^{ \pm}, \ldots, s_{N-1}^{ \pm}\right\}$and $\mathbf{s}^{ \pm}=\left\{s_{0}^{ \pm}, s_{1}^{ \pm}, \ldots, s_{N-1}^{ \pm}, s_{N}^{ \pm}\right\}$. The subsystem evolution and the IFs are now given by 


$$
\begin{aligned}
I_{S}\left(\mathbf{s}^{\prime \pm}\right) & =\left\langle s_{0}^{+}\left|\rho_{S}(0)\right| s_{0}^{-}\right\rangle K\left(s_{N}^{ \pm}, s_{N-1}^{ \pm}\right) \ldots K\left(s_{2}^{ \pm}, s_{1}^{ \pm}\right) K\left(s_{1}^{ \pm}, s_{0}^{ \pm}\right) \\
I_{B}\left(\mathbf{s}^{\prime \pm}\right) & =\operatorname{Tr}_{B}\left[e^{-i W_{B}\left(s_{N}^{+}\right) \delta t / 2} e^{-i W_{B}\left(s_{N-1}^{+}\right) \delta t} \ldots e^{-i W_{B}\left(s_{0}^{+}\right) \delta t / 2} \rho_{B} e^{i W_{B}\left(s_{0}^{-}\right) \delta t / 2} \ldots e^{i W_{B}\left(s_{N-1}^{-}\right) \delta t} e^{i W_{B}\left(s_{N}^{-}\right) \delta t / 2}\right] \\
I_{F}\left(\mathbf{s}^{\prime \pm}\right) & =\operatorname{Tr}_{F}\left[e^{-i W_{F}\left(s_{N}^{+}\right) \delta t / 2} e^{-i W_{F}\left(s_{N-1}^{+}\right) \delta t} \ldots e^{-i W_{F}\left(s_{0}^{+}\right) \delta t / 2} \rho_{F} e^{i W_{F}\left(s_{0}^{-}\right) \delta t / 2} \ldots e^{i W_{F}\left(s_{N-1}^{-}\right) \delta t} e^{i W_{F}\left(s_{N}^{-}\right) \delta t / 2}\right]
\end{aligned}
$$

where

$$
K\left(s_{k+1}^{ \pm}, s_{k}^{ \pm}\right)=\left\langle s_{k+1}^{+}\left|e^{-i H_{S} \delta t}\right| s_{k}^{+}\right\rangle\left\langle s_{k}^{-}\left|e^{i H_{S} \delta t}\right| s_{k+1}^{-}\right\rangle
$$

is the propagator matrix for the subsystem. We have also used the short notation $W$ for bath operators that are evaluated along the path,

$$
\begin{aligned}
& W_{F}(s)=H_{F}+\left\langle s\left|V_{S F}\right| s\right\rangle, \\
& W_{B}(s)=H_{B}+\left\langle s\left|V_{S B}\right| s\right\rangle .
\end{aligned}
$$

In the next sections we explain how we compute the bosonic and fermionic IFs. The former has a closed analytic form in certain situations. The latter is computed only numerically.

\section{B. Bosonic IF}

We present the structure of the bosonic IF in two separate models, corresponding to different types of subsystem-boson bath interactions. In both cases the bosonic bath is prepared in a canonical state of inverse temperature $\beta_{p h}=1 / T_{p h}$,

$$
\rho_{B}=e^{-\beta_{p h} H_{B}} / \operatorname{Tr}_{B}\left[e^{-\beta_{p h} H_{B}}\right]
$$

Displacement interaction model, $v_{p, p^{\prime}}^{B}=0$ and $\zeta_{p, p^{\prime}}^{B}=0$. Given the remaining linear displacement-polarization interaction, an analytic form for the bosonic IF can be written, the so-called "Feynman-Vernon" influence functional (FV IF) [33]. In its time-discrete form, the bosonic IF is given by an exponent with pairwise interactions along the path [16]

$$
I_{B}\left(s_{0}^{ \pm}, \ldots, s_{N}^{ \pm}\right)=\exp \left[-\sum_{k=0}^{N} \sum_{k^{\prime}=0}^{k}\left(s_{k}^{+}-s_{k}^{-}\right)\left(\eta_{k, k^{\prime}} s_{k^{\prime}}^{+}-\eta_{k, k^{\prime}}^{*} s_{k^{\prime}}^{-}\right)\right] .
$$

The coefficients $\eta_{k, k^{\prime}}$ are additive in the number of thermal baths, and they depend on these baths' spectral functions and initial temperatures [16]. For completeness, these coefficients are included in Appendix A. 
Boson scattering model, $\xi_{p}^{B}=0$. The bosonic IF can now be computed numerically, by using the trace formula for bosons [34]

$$
\operatorname{Tr}_{B}\left[e^{M_{1}} e^{M_{2}} \ldots e^{M_{k}}\right]=\operatorname{det}\left[1-e^{m_{1}} e^{m_{2}} \ldots e^{m_{k}}\right]^{-1} .
$$

Here $m_{k}$ is a single particle operator corresponding to a quadratic bosonic operator $M_{k}=$ $\sum_{p, p^{\prime}}\left(m_{k}\right)_{p, p^{\prime}} b_{p}^{\dagger} b_{p^{\prime}}$. Application of the trace formula to the bosonic IF (13) leads to

$$
\begin{aligned}
I_{B} & =\operatorname{Tr}_{B}\left[e^{M_{1}} e^{M_{2}} \ldots e^{M_{k}} \rho_{B}\right] \\
& =\operatorname{det}\left\{\left[\hat{I}_{B}+f_{B}\right]-e^{m_{1}} e^{m_{2}} \ldots e^{m_{k}} f_{B}\right\}^{-1} .
\end{aligned}
$$

The matrix $\hat{I}_{B}$ is an identity matrix, and the function $f_{B}$ stands for the Bose-Einstein distribution, $f_{B}=\left[e^{\beta_{p h} \omega}-1\right]^{-1}$. The determinant in Eq. (19) can be evaluated numerically by taking into account $L_{B}$ modes for the boson bath. This discretization implies a numerical error. Generalizations, to include more that one bosonic baths, are immediate.

\section{Fermionic IF}

The fermionic IF is computed numerically since an exact analytic form is not known in the general strong coupling limit [29-31]. It is calculated by using the trace formula for fermions [34]

$$
\operatorname{Tr}_{F}\left[e^{M_{1}} e^{M_{2}} \ldots e^{M_{k}}\right]=\operatorname{det}\left[1+e^{m_{1}} e^{m_{2}} \ldots e^{m_{k}}\right]
$$

Here $m_{k}$ is a single particle operator corresponding to a quadratic operator $M_{k}=\sum_{i, j}\left(m_{k}\right)_{i, j} c_{i}^{\dagger} c_{j}$. In the next section we consider a model with two Fermi seas, $H_{F}=H_{L}+H_{R}$, prepared in a factorized state of distinct grand canonical states, $\rho_{F}=\rho_{L} \otimes \rho_{R}$, with

$$
\rho_{\nu}=e^{-\beta_{\nu}\left(H_{\nu}-\mu_{\nu} N_{\nu}\right)} / \operatorname{Tr}_{F}\left[e^{-\beta_{\nu}\left(H_{\nu}-\mu_{\nu} N_{\nu}\right)}\right], \quad \nu=L, R
$$

Here $\beta_{\nu}=1 / T_{\nu}$ stands for an inverse temperature, and $\mu_{\nu}$ denotes the chemical potential of the $\nu$ bath. Application of the trace formula to the fermionic IF in Eq. (13) leads to

$$
\begin{aligned}
I_{F} & =\operatorname{Tr}_{F}\left[e^{M_{1}} e^{M_{2}} \ldots e^{M_{k}} \rho_{F}\right] \\
& =\operatorname{det}\left\{\left[\hat{I}_{L}-f_{L}\right] \otimes\left[\hat{I}_{R}-f_{R}\right]+e^{m_{1}} e^{m_{2}} \ldots e^{m_{k}}\left[f_{L} \otimes f_{R}\right]\right\} .
\end{aligned}
$$

The matrices $\hat{I}_{\nu}$ are the identity matrices for the $\nu=L, R$ space. The functions $f_{L}$ and $f_{R}$ are the bands electrons' energy distribution, $f_{\nu}=\left[e^{\beta_{\nu}\left(\epsilon-\mu_{\nu}\right)}+1\right]^{-1}$. The determinant in Eq. (22) can be evaluated numerically by taking into account $L_{s}$ electronic states for each metal. This discretization implies a numerical error. 


\section{The iterative scheme}

The dynamics described by Equation (12) includes long-range interactions along the path, limiting brute force direct numerical simulations to very short times. The iterative scheme, developed in Ref. [17], is based on the observation that in standard nonequilibrium situations and at finite temperatures bath correlations exponentially die [24, 31], thus the IF can be truncated beyond a memory time $\tau_{c}=N_{s} \delta t$, corresponding to the time where bath correlations sustain. Here $N_{s}$ is an integer, $\delta t$ is the discretized time step, and the correlation time $\tau_{c}$ is dictated by the bias and temperature. Roughly, for a system under a potential bias $\Delta \mu$ and a temperature $T, \tau_{c} \sim \max \{1 / T, 1 / \Delta \mu\}$ [17]. By recursively breaking the IF to include terms only within $\tau_{c}$, we reach the following (non-unique) structure for the $\alpha=B, F$ influence functional,

$$
\begin{aligned}
& I_{\alpha}\left(s_{0}^{ \pm}, s_{1}^{ \pm}, s_{2}^{ \pm}, \ldots, s_{N}^{ \pm}\right) \approx I_{\alpha}\left(s_{0}^{ \pm}, s_{1}^{ \pm}, \ldots, s_{N_{s}}^{ \pm}\right) I_{\alpha}^{\left(N_{s}\right)}\left(s_{1}^{ \pm}, s_{2}^{ \pm}, \ldots, s_{N_{s}+1}^{ \pm}\right) I_{\alpha}^{\left(N_{s}\right)}\left(s_{2}^{ \pm}, s_{3}^{ \pm}, \ldots, s_{N_{s}+2}^{ \pm}\right) \ldots \\
& \times I_{\alpha}^{\left(N_{s}\right)}\left(s_{N-N_{s}}^{ \pm}, s_{N-N_{s}+1}^{ \pm}, \ldots, s_{N}^{ \pm}\right)
\end{aligned}
$$

where we identify the "truncated IF", $I_{\alpha}^{\left(N_{s}\right)}$, as the ratio between two IFs, with the numerator calculated with an additional time step,

$$
I_{\alpha}^{\left(N_{s}\right)}\left(s_{k}, s_{k+1}, \ldots, s_{k+N_{s}}\right)=\frac{I_{\alpha}\left(s_{k}^{ \pm}, s_{k+1}^{ \pm}, \ldots, s_{k+N_{s}}^{ \pm}\right)}{I_{\alpha}\left(s_{k}^{ \pm}, s_{k+1}^{ \pm}, \ldots, s_{k+N_{s}-1}^{ \pm}\right)} .
$$

The truncated IF is the central object in our calculations. For fermions, its numerator and denominator are separately computed using Eq. (22). The bosonic IF is similarly computed with the help of Eq. (19) when $\xi_{p}^{B}=0$. In the complementary case, $\zeta_{p, p^{\prime}}^{B}=0$ and $v_{p, p^{\prime}}^{B}=0$, the truncated-bosonic IF has a closed analytic form: Using Eq. (17) we find that it comprises only two-body interactions, of $s_{k+N_{s}}$ with the preceding spins, down to $s_{k}$,

$$
I_{B}^{\left(N_{s}\right)}\left(s_{k}, s_{k+1}, \ldots, s_{k+N_{s}}\right)=\exp \left[-\sum_{k^{\prime}=k}^{k+N_{s}}\left(s_{k+N_{s}}^{+}-s_{k+N_{s}}^{-}\right)\left(\eta_{k+N_{s}, k^{\prime}} s_{k^{\prime}}^{+}-\eta_{k+N_{s}, k^{\prime}}^{*} s_{k^{\prime}}^{-}\right)\right] .
$$

Based on the decompositions (24) and (25), we time-evolve Eq. (12) iteratively, by defining a multi-time reduced density matrix $\tilde{\rho}_{S}\left(s_{k}, s_{k+1}, . ., s_{k+N_{s}-1}\right)$. Its initial value is given by

$$
\tilde{\rho}_{S}\left(s_{0}^{ \pm}, \ldots, s_{N_{s}}^{ \pm}\right)=I_{S}\left(s_{0}^{ \pm}, \ldots, s_{N_{s}}^{ \pm}\right) I_{B}\left(s_{0}^{ \pm}, \ldots, s_{N_{s}}^{ \pm}\right) I_{F}\left(s_{0}^{ \pm}, \ldots, s_{N_{s}}^{ \pm}\right) .
$$


Its evolution is dictated by

$$
\begin{aligned}
& \tilde{\rho}_{S}\left(s_{k+1}^{ \pm}, \ldots, s_{k+N_{s}}^{ \pm}\right)=\sum_{s_{k}^{ \pm}} \tilde{\rho}_{S}\left(s_{k}^{ \pm}, \ldots, s_{k+N_{s}-1}^{ \pm}\right) K\left(s_{k+N_{s}}^{ \pm}, s_{k+N_{s}-1}^{ \pm}\right) \\
& \times I_{F}^{\left(N_{s}\right)}\left(s_{k}^{ \pm}, \ldots, s_{k+N_{s}}^{ \pm}\right) I_{B}^{\left(N_{s}\right)}\left(s_{k}^{ \pm}, \ldots, s_{k+N_{s}}^{ \pm}\right) .
\end{aligned}
$$

The time-local $\left(t_{k}=k \delta t\right)$ reduced density matrix, describing the state of the subsystem at a certain time, is reached by summing over all intermediate states,

$$
\rho_{S}\left(t_{k}\right)=\sum_{s_{k-1}^{ \pm} \ldots s_{k-N_{s}+1}^{ \pm}} \tilde{\rho}_{S}\left(s_{k-N_{s}+1}^{ \pm}, \ldots, s_{k}^{ \pm}\right) .
$$

The bosonic and fermionic IFs may be (and often this is the case) characterized by different memory time. Thus, in principle we could truncate the fermionic IF to include $N_{s}^{F}$ terms, and the bosonic IF to include $N_{s}^{B}$ elements. However, the efficiency of the computation is dictated by the longest memory time, thus, for convenience, we truncate both IFs using the largest value, identified by $N_{s}$.

By construction, this iterative approach conserves the trace of the reduced density matrix, ensuring the stability of the iterative algorithm to long times [16]. This property can be inferred from Eqs. (12) and (13), by using the formal expressions for the truncated IFs, Eq. (23) and (24). To prover this property, we trace over the reduced density matrix at time $t$, identifying $s_{N}=s_{N}^{+}=s_{N}^{-}$,

$$
\begin{aligned}
\operatorname{Tr}_{S}\left[\rho_{S}(t)\right] & \equiv \sum_{s_{N}}\left\langle s_{N}\left|\rho_{S}(t)\right| s_{N}\right\rangle \\
& =\sum_{\mathbf{s}^{\prime \pm}} I_{S}\left(\mathbf{s}^{\prime \pm}\right) I_{F}\left(\mathbf{s}^{ \pm}\right) I_{B}\left(\mathbf{s}^{\prime \pm}\right) \delta\left(s_{N}^{+}-s_{N}^{-}\right)
\end{aligned}
$$

Using the cyclic property of the trace, we note that both the fermionic and bosonic IFs are independent of $s_{N}$, when $s_{N}^{+}=s_{N}^{-}$. Therefore, the summation over the $s_{N}$ coordinate reduces to a simple sum which can be performed using the completeness relation for the subsystem states, resulting in

$$
\sum_{s_{N}}\left\langle s_{N}\left|e^{-i H_{S} \delta t}\right| s_{N-1}^{+}\right\rangle\left\langle s_{N-1}^{-}\left|e^{i H_{S} \delta t}\right| s_{N}\right\rangle=\delta\left(s_{N-1}^{+}-s_{N-1}^{-}\right)
$$


Iterating in this manner we conclude that

$$
\begin{aligned}
\operatorname{Tr}_{S}\left[\rho_{S}(t)\right] & \equiv \sum_{s_{N}}\left\langle s_{N}\left|\rho_{S}(t)\right| s_{N}\right\rangle \\
& =\sum_{\mathbf{s}^{ \pm}} I_{S}\left(\mathbf{s}^{\prime \pm}\right) I_{F}\left(\mathbf{s}^{\prime \pm}\right) I_{B}\left(\mathbf{s}^{\prime \pm}\right) \delta\left(s_{N}^{+}-s_{N}^{-}\right) \delta\left(s_{N-1}^{+}-s_{N-1}^{-}\right) \ldots \delta\left(s_{1}^{+}-s_{1}^{-}\right) \delta\left(s_{0}^{+}-s_{0}^{-}\right) \\
& =\sum_{s_{0}}\left\langle s_{0}\left|\rho_{S}(0)\right| s_{0}\right\rangle=\operatorname{Tr}_{S}\left[\rho_{S}(0)\right]
\end{aligned}
$$

We emphasize that the trace conservation is maintained even with the use of the truncated form for the IFs. Moreover, it holds irrespective of the details of the bath and the systembath interaction form. It is also obeyed in the more general case, Eq. (91). Equation (27) [and its generalized form, Eq. (34) below], describe a linear map. Its fixed points are stable if the eigenvalues of the map have modulus less than one, which is the case here. Thus, our scheme is expected to approach a stationary-state in the long time limit.

\section{E. Expectation values for operators}

Besides the reduced density matrix, we can also acquire the time evolution of several expectation values. Adopting the Hamiltonian (11), we illustrate next how we achieve the charge current behavior. For simplicity, we consider the case with only two fermionic reservoirs, $\nu=L, R$. The current operator, e.g., at the $L$ bath is defined as the time derivative of the number operator. The expectation value of this current is given by

$$
j_{L}=-\frac{d}{d t} \operatorname{Tr}\left[\rho N_{L}\right], \quad N_{L} \equiv \sum_{j \in L} c_{j}^{\dagger} c_{j}
$$

We consider the time evolution of the related exponential operator $e^{\lambda N_{L}}$, with $\lambda$ a real number that is taken to vanish at the end of the calculation,

$$
\begin{aligned}
\left\langle N_{L}(t)\right\rangle & \equiv \operatorname{Tr}\left[\rho N_{L}(t)\right] \\
& =\lim _{\lambda \rightarrow 0} \frac{\partial}{\partial \lambda} \operatorname{Tr}\left[\rho(0) e^{i H t} e^{\lambda N_{L}} e^{-i H t}\right] .
\end{aligned}
$$

As before, the initial condition is factorized at $t=0, \rho(0)=\rho_{S}(0) \otimes \rho_{B} \otimes \rho_{F}$. The trace is performed over subsystem and reservoirs degrees of freedom. By following the same steps as in Eqs. (3)-(17), we reach the path-integral expression 


$$
\begin{aligned}
& \quad\left\langle e^{\lambda N_{L}(t)}\right\rangle=\sum_{s_{0}^{ \pm}} \sum_{s_{1}^{ \pm}} \ldots \sum_{s_{N-1}^{ \pm}} \sum_{s_{N}} \operatorname{Tr}_{B} \operatorname{Tr}_{F}\left[e^{\lambda N_{L}}\left\langle s_{N}\left|\mathcal{G}^{\dagger}\right| s_{N-1}^{+}\right\rangle\left\langle s_{N-1}^{+}\left|\mathcal{G}^{\dagger}\right| s_{N-2}^{+}\right\rangle \ldots\right. \\
& \left.\times\left\langle s_{0}^{+}|\rho(0)| s_{0}^{-}\right\rangle \ldots\left\langle s_{N-2}^{-}|\mathcal{G}| s_{N-1}^{-}\right\rangle\left\langle s_{N-1}^{-}|\mathcal{G}| s_{N}\right\rangle\right] .
\end{aligned}
$$

Factorizing the time evolution operators using Eq. (5), we accomplish the compact form

$$
\left\langle e^{\lambda N_{L}(t)}\right\rangle=\sum_{\mathbf{s}^{\prime \pm}} I_{S}\left(\mathbf{s}^{\prime \pm}\right) I_{B}\left(\mathbf{s}^{\prime \pm}\right) \tilde{I}_{F}\left(\mathbf{s}^{\prime \pm}\right) \delta\left(s_{N}^{+}-s_{N}^{-}\right)
$$

The terms $I_{S}$ and $I_{B}$ are given in Eq. (13). The fermionic IF accommodates an additional exponent,

$\tilde{I}_{F}\left(\mathbf{s}^{ \pm}\right)=\operatorname{Tr}_{F}\left[e^{\lambda N_{L}} e^{-i W_{F}\left(s_{N}^{+}\right) \delta t / 2} e^{-i W_{F}\left(s_{N-1}^{+}\right) \delta t} \ldots e^{-i W_{F}\left(s_{0}^{+}\right) \delta t / 2} \rho_{F} e^{i W_{F}\left(s_{0}^{-}\right) \delta t / 2} \ldots . e^{i W_{F}\left(s_{N-1}^{-}\right) \delta t} e^{i W_{F}\left(s_{N}^{-}\right) \delta t / 2}\right]$.

We can time evolve the operator $\left\langle e^{\lambda N_{L}}\right\rangle$ by using the iterative scheme of Sec. III.D, by truncating the bosonic and fermionic IFs up to the memory time $\tau_{c}=N_{s} \delta t$, for several values of $\lambda$. We then take the numerical derivative with respect to $\lambda$ and $t$, to attain the charge current itself.

The approach explained here could be used to explore several fermionic operators, for example, the averaged current $j_{a v}=\left(j_{L}-j_{R}\right) / 2$. The minus sign in front of $j_{R}$ originates from the sign notation, with the current defined positive when flowing $L$ to $R$. The implementation of a heat current operator, describing the heat current flowing between two bosonic reservoirs, requires first the derivation of an analytic form for the bosonic IF, an expression analogous to the FV IF, and the subsequent time discretization of this IF, to reach an expression analogous to (17).

\section{F. Expression for multilevel subsystems and general interactions}

So far we have detailed the iterative time evolution scheme for the spin-boson-fermion model (11). The procedure can be extended, to treat more complex cases. Based on the general principles outlined in Sec. IIID, one notes that the path-integral expression (9) can be evaluated iteratively by generalizing Eq. (27) to the form

$$
\begin{aligned}
& \tilde{\rho}_{S}\left(v_{k+1}^{ \pm}, \ldots, v_{k+N_{s}}^{ \pm}\right)= \\
& \sum_{v_{k}^{ \pm}} \tilde{\rho}_{S}\left(v_{k}^{ \pm}, \ldots, v_{k+N_{s}-1}^{ \pm}\right) K\left(m_{k+N_{s}}^{ \pm}, n_{k+N_{s}}^{ \pm}\right) I_{F}^{\left(N_{S}\right)}\left(s_{k}^{ \pm}, f_{k}^{ \pm}, g_{k}^{ \pm}, \ldots, s_{k+N_{s}}^{ \pm}, f_{k+N_{s}}^{ \pm}, g_{k+N_{s}}^{ \pm}\right) \\
& \times I_{B}^{\left(N_{s}\right)}\left(f_{k}^{ \pm}, g_{k}^{ \pm}, m_{k}^{ \pm}, n_{k}^{ \pm}, \ldots, f_{k+N_{s}}^{ \pm}, g_{k+N_{s}}^{ \pm}, m_{k+N_{s}}^{ \pm}, n_{k+N_{s}}^{ \pm}\right)
\end{aligned}
$$


where we compact several variables, $v_{k}^{ \pm}=\left\{s_{k}^{ \pm}, f_{k}^{ \pm}, g_{k}^{ \pm}, m_{k}^{ \pm}, n_{k}^{ \pm}\right\}$. It should be noted that in cases when the IF is time invariant, as in the molecular electronics case discussed below, one needs to evaluate $I_{B}^{\left(N_{s}\right)}$ and $I_{F}^{\left(N_{s}\right)}$ only once, then use the saved array to time-evolve the auxiliary density matrix.

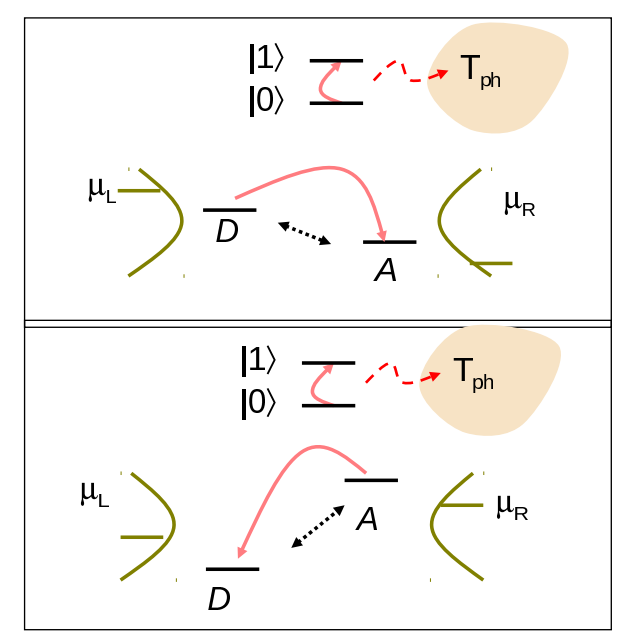

FIG. 2. Molecular electronic rectifier setup. A biased donor-acceptor electronic junction is coupled to an anharmonic mode, represented by the two-state system with vibrational levels $|0\rangle$ and $|1\rangle$. This molecular vibrational mode may further relax its energy to a phononic thermal reservoir. This process is represented by a dashed arrow. Direct electron tunneling element between D and A is depicted by a dotted double arrow. Top: $\Delta \mu>0$. In our construction both molecular electronic levels are placed within the bias window at large positive bias, resulting in a large (resonant) current. Bottom: At negative bias the energy of $\mathrm{A}$ is placed outside the bias window, thus the total charge current is small.

\section{APPLICATION: MOLECULAR RECTIFIER}

The functionality and stability of electron-conducting molecular junctions are directly linked to heating and cooling effects experienced by molecular vibrational modes in biased situations [4, 35-41]. In particular, junction heating and breakdown may occur once the bias voltage exceeds typical molecular vibrational frequencies, when the electronic levels are situated within the bias window, if energy dissipation from the molecule to its environment is not efficient. 
In this section we study the dynamics and steady-state behavior of electrons and a specific vibrational mode in a molecular conducting junction serving as an electrical rectifier. The rectifier Hamiltonian is detailed in Sec. IVA. In Sec. IVB we show that this model can be mapped onto the spin-boson-fermion Hamiltonian (11). This allows us to employ the path-integral technique of Sec. III for simulating the rectifier dynamics. The rectification mechanism is explained in Sec. IVC. Relevant expressions of a (perturbative) Master equation method are described in Sec. IVD, to be compared to our path-integral based results in Sec. IVE, Convergence issues and computational aspects are discussed in Sec. IVF.

\section{A. Rectifier Hamiltonian}

The D-A rectifier model includes a biased molecular electronic junction and a selected (generally anharmonic) internal vibrational mode which is coupled to an electronic transition in the junction and to a secondary phonon bath, representing other molecular and environmental degrees of freedom. In the present study we model the anharmonic mode by a two-state system, and this model can already capture the essence of the vibrational instability effect [27]. For a schematic representation, see Fig. 2. This model allows us to investigate the exchange of electronic energy with molecular vibrational heating, and the competition between elastic and inelastic transport mechanisms. Its close variant has been adopted in Refs. [42 44] for studying the thermopower and thermal transport of electrons in molecular junctions with electron-phonon interactions, within the linear response regime.

We assume that the D molecular group is strongly attached to the neighboring $L$ metal surface, and that this unit is overall characterized by the chemical potential $\mu_{L}$. Similarly, the A group is connected to the metal $R$, characterized by $\mu_{R}$. At time $t=0$ the $\mathrm{D}$ and A states are put into contact. Experimentally, the $R$ metal may stand for an STM tip decorated by a molecular group. This tip is approaching the D site which is attached to the metal surface $L$. Once the D and A molecular groups are put into contact, electrons can flow across the junction in two parallel pathways: (i) through a direct D-A tunneling mechanism, and (ii) inelastically, assisted by a vibration: excess electron energy goes to excite the D-A vibrational motion, and vice versa.

The rectifier (rec) Hamiltonian includes the electronic Hamiltonian $H_{e l}$ with decoupled D and A states, the vibrational, two-state subsystem $H_{v i b}$, electronic-vibrational coupling 
$H_{I}$, a free phonon Hamiltonian $H_{p h}$, and the coupling of this secondary phonon bath to the selected vibration,

$$
\bar{H}_{r e c}=H_{e l}+H_{v i b}+H_{I}+H_{p h}+H_{v i b-p h} .
$$

The electronic (fermionic) contribution $H_{e l}$ attends for all fermionic terms besides the direct D and A tunneling term, which for convenience is included in $H_{I}$,

$$
\begin{aligned}
H_{e l} & =H_{M}+H_{L}^{0}+H_{R}^{0}+H_{C} \\
H_{M} & =\epsilon_{d} c_{d}^{\dagger} c_{d}+\epsilon_{a} c_{a}^{\dagger} c_{a} \\
H_{L}^{0} & =\sum_{l \in L} \epsilon_{l} c_{l}^{\dagger} c_{l} ; \quad H_{R}^{0}=\sum_{r \in R} \epsilon_{r} c_{r}^{\dagger} c_{r} . \\
H_{C} & =\sum_{l} v_{l}\left(c_{l}^{\dagger} c_{d}+c_{d}^{\dagger} c_{l}\right)+\sum_{r} v_{r}\left(c_{r}^{\dagger} c_{a}+c_{a}^{\dagger} c_{r}\right) .
\end{aligned}
$$

$H_{M}$ stands for the molecular electronic part including two electronic states, a donor $\mathrm{D}$ and an acceptor A. $c_{d / a}^{\dagger}\left(c_{d / a}\right)$ is a fermionic creation (annihilation) operator of an electron on the $\mathrm{D}$ or A sites, of energies $\epsilon_{d, a}$. The two metals, $H_{\nu}^{0}, \nu=L, R$, are each composed of a collection of noninteracting electrons. The hybridization of the $\mathrm{D}$ state to the left $(L)$ bath, and similarly, the coupling of the A site to the right $(R)$ metal, are described by $H_{C}$. The vibrational Hamiltonian includes a special nuclear anharmonic vibrational mode of frequency $\omega_{0}$,

$$
H_{v i b}=\frac{\omega_{0}}{2} \sigma_{z}
$$

The displacement of this mode from equilibrium is coupled to an electron transition in the system, with an energy cost $\kappa$, resulting in heating and/or cooling effects,

$$
H_{I}=\left(\kappa \sigma_{x}+v_{d a}\right)\left(c_{d}^{\dagger} c_{a}+c_{a}^{\dagger} c_{d}\right) .
$$

Besides the electron-vibration coupling term, $H_{I}$ further includes a direct electron tunneling element between the $\mathrm{D}$ and the $\mathrm{A}$ states, of strength $v_{d a}$. Electron transfer between the two metals can therefore proceed through two mechanisms: coherent tunneling and vibrationalassisted inelastic transport.

The selected vibrational mode may couple to many other phonons, either internal to the molecules or external, grouped into a harmonic reservoir,

$$
\begin{aligned}
H_{p h} & =\sum_{p} \omega_{p} b_{p}^{\dagger} b_{p} \\
H_{v i b-p h} & =\sigma_{x} \sum_{p} \xi_{p}^{B}\left(b_{p}^{\dagger}+b_{p}\right)
\end{aligned}
$$


The Hamiltonian $H_{v i b-p h}$ corresponds to a displacement-displacement interaction type.

The motivation behind the choice of the two-level system (TLS) mode is twofold. First, as we showed in Ref. [27], the development of vibrational instability in the D-A rectifier does not depend on the mode harmonicity, at least in the weak electron-phonon coupling limit. Since it is easier to simulate a truncated mode with our approach, rather than a harmonic mode, we settle on the TLS model. Second, while there are many studies where a perfectly harmonic mode is assumed, for example, see Refs. [20, 21, 24], to the best of our knowledge our work is the first to explore electron conduction in the limit of strong vibrational anharmonicity.

\section{B. Mapping to the spin-boson-fermion model}

We diagonalize the electronic part of the Hamiltonian $H_{e l}$ to acquire, separately, the exact eigenstates for the $L$-half and $R$-half ends of $H_{e l}$,

$$
\begin{aligned}
H_{e l} & =H_{L}+H_{R} \\
H_{L} & =\sum_{l} \epsilon_{l} a_{l}^{\dagger} a_{l}, \quad H_{R}=\sum_{r} \epsilon_{r} a_{r}^{\dagger} a_{r} .
\end{aligned}
$$

Assuming that the reservoirs are dense, their new operators are assigned energies that are the same as those before diagonalization. The D and A (new) energies are assumed to be placed within a band of continuous states, excluding the existence of bound states. The old operators are related to the new ones by [45]

$$
\begin{aligned}
c_{d} & =\sum_{l} \lambda_{l} a_{l}, & c_{l} & =\sum_{l^{\prime}} \eta_{l, l^{\prime}} a_{l^{\prime}} \\
c_{a} & =\sum_{r} \lambda_{r} a_{r}, & c_{r} & =\sum_{r^{\prime}} \eta_{r, r^{\prime}} a_{r^{\prime}},
\end{aligned}
$$

where the coefficients, e.g., for the $L$ set, are given by

$$
\begin{aligned}
\lambda_{l} & =\frac{v_{l}}{\epsilon_{l}-\epsilon_{d}-\sum_{l^{\prime}} \frac{v_{l^{\prime}}^{2}}{\epsilon_{l}-\epsilon_{l^{\prime}}+i \delta}} \\
\eta_{l, l^{\prime}} & =\delta_{l, l^{\prime}}-\frac{v_{l} \lambda_{l^{\prime}}}{\epsilon_{l}-\epsilon_{l^{\prime}}+i \delta} .
\end{aligned}
$$

Similar expressions hold for the $R$ set. It is easy to derive the following relation,

$$
\sum_{l^{\prime}} \frac{v_{l^{\prime}}^{2}}{\epsilon_{l}-\epsilon_{l^{\prime}}+i \delta}=P P \sum_{l^{\prime}} \frac{v_{l^{\prime}}^{2}}{\epsilon_{l}-\epsilon_{l^{\prime}}}-i \Gamma_{L}\left(\epsilon_{l}\right) / 2,
$$


with the hybridization strength $\left(v_{j}\right.$ is assumed real),

$$
\Gamma_{L}(\epsilon)=2 \pi \sum_{l} v_{l}^{2} \delta\left(\epsilon-\epsilon_{l}\right)
$$

With the new operators, the Hamiltonian (35) can be rewritten as

$$
\begin{aligned}
\bar{H}_{r e c} & =\sum_{l} \epsilon_{l} a_{l}^{\dagger} a_{l}+\sum_{r} \epsilon_{r} a_{r}^{\dagger} a_{r}+\frac{\omega_{0}}{2} \sigma_{z} \\
& +\left(\kappa \sigma_{x}+v_{d a}\right) \sum_{l, r}\left[\lambda_{l}^{*} \lambda_{r} a_{l}^{\dagger} a_{r}+\lambda_{r}^{*} \lambda_{l} a_{r}^{\dagger} a_{l}\right] \\
& +\sum_{p} \omega_{p} b_{p}^{\dagger} b_{p}+\sigma_{x} \sum_{p} \xi_{p}^{B}\left(b_{p}^{\dagger}+b_{p}\right) .
\end{aligned}
$$

This Hamiltonian can be transformed into the spin-boson-fermion model of zero energy spacing, using the unitary transformation

$$
U^{\dagger} \sigma_{z} U=\sigma_{x}, \quad U^{\dagger} \sigma_{x} U=\sigma_{z}
$$

with $U=\frac{1}{\sqrt{2}}\left(\sigma_{x}+\sigma_{z}\right)$. The transformed Hamiltonian $H_{r e c}=U^{\dagger} \bar{H}_{r e c} U$ includes a $\sigma_{z}$-type electron-vibration coupling,

$$
\begin{aligned}
H_{r e c} & =\sum_{l} \epsilon_{l} a_{l}^{\dagger} a_{l}+\sum_{r} \epsilon_{r} a_{r}^{\dagger} a_{r}+\frac{\omega_{0}}{2} \sigma_{x} \\
& +\left(\kappa \sigma_{z}+v_{d a}\right) \sum_{l, r}\left[\lambda_{l}^{*} \lambda_{r} a_{l}^{\dagger} a_{r}+\lambda_{r}^{*} \lambda_{l} a_{r}^{\dagger} a_{l}\right] \\
& +\sum_{p} \omega_{p} b_{p}^{\dagger} b_{p}+\sigma_{z} \sum_{p} \xi_{p}^{B}\left(b_{p}^{\dagger}+b_{p}\right) .
\end{aligned}
$$

It describes a spin (TLS) coupled diagonally to two fermionic environments and to a single boson bath. One can immediately confirm that this Hamiltonian is accounted for by Eq. (11). To simplify our notation, we further identify the electronic-vibration effective coupling parameter

$$
\xi_{l, r}^{F}=\kappa \lambda_{l}^{*} \lambda_{r}
$$

For later use we also define the spectral function of the secondary phonon bath as

$$
J_{p h}(\omega)=\pi \sum_{p}\left(\xi_{p}^{B}\right)^{2} \delta\left(\omega-\omega_{p}\right) .
$$

In our simulations below we adopt an ohmic function,

$$
J_{p h}(\omega)=\frac{\pi K_{d}}{2} \omega e^{-\omega / \omega_{c}}
$$


with the dimensionless Kondo parameter $K_{d}$, characterizing subsystem-bath coupling, and the cutoff frequency $\omega_{c}$.

As an initial condition for the reservoirs, we assume canonical distributions with the boson-phonon bath distribution following $\rho_{B}=e^{-\beta_{p h} H_{p h}} / \operatorname{Tr}_{B}\left[e^{-\beta_{p h} H_{p h}}\right]$ and the electronicfermionic initial density matrix obeying $\rho_{F}=\rho_{L} \otimes \rho_{R}$, with $\rho_{\nu}=e^{-\beta_{\nu}\left(H_{\nu}-\mu_{\nu} N_{\nu}\right)} / \operatorname{Tr}_{F}\left[e^{-\beta_{\nu}\left(H_{\nu}-\mu_{\nu} N_{\nu}\right)}\right]$, $\nu=L, R$. This results in the expectation values of the exact eigenstates,

$$
\left\langle a_{l}^{\dagger} a_{l^{\prime}}\right\rangle=\delta_{l, l^{\prime}} f_{L}\left(\epsilon_{l}\right), \quad\left\langle a_{r}^{\dagger} a_{r^{\prime}}\right\rangle=\delta_{r, r^{\prime}} f_{R}\left(\epsilon_{r}\right)
$$

where $f_{L}(\epsilon)=\left[\exp \left(\beta_{L}\left(\epsilon-\mu_{L}\right)\right)+1\right]^{-1}$ denotes the Fermi distribution function. An analogous expression holds for $f_{R}(\epsilon)$. The reservoirs temperatures are denoted by $1 / \beta_{\nu}$; the chemical potentials are $\mu_{\nu}$.
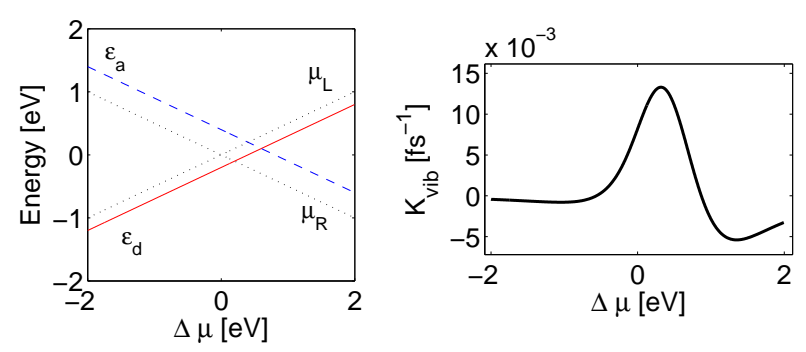

FIG. 3. Left panel: Energy of the donor (full line) and acceptor states (dashed line). The dotted lines correspond to the chemical potentials at the left and right sides. Right panel: Damping rate $K_{v i b}$. The junction's parameters are $\Gamma_{\nu}=1, \beta_{\nu}=200, \kappa=0.1, \omega_{0}=0.2$, and $\epsilon_{d}(\Delta \mu=0)=-0.2$, $\epsilon_{a}(\Delta \mu=0)=0.4$. We used fermionic metals with a linear dispersion relations for the original $H_{\nu}^{0}$ baths and sharp cutoffs at \pm 1 . All energy parameters are given in units of $\mathrm{eV}$.

\section{Rectifying mechanism}

We now explain the operation principles of the molecular rectifier. In our construction the application of a bias voltage linearly shifts the energies of the molecular electronic levels, D and $\mathrm{A}$. In equilibrium, we set $\epsilon_{a}<0$ and $\epsilon_{d}>0$. Under positive bias, defined as $\mu_{L}-\mu_{R}>0$, the energy of the acceptor level increases, and the donor level drops down, see Fig. 2, When both levels are buried within the bias window, the junction can support large currents. At negative bias the electronic level $\mathrm{A}$ is positioned above the bias window, resulting in small currents. For a scheme of the energy organization of the system, see Fig. 3 panel, left panel. 

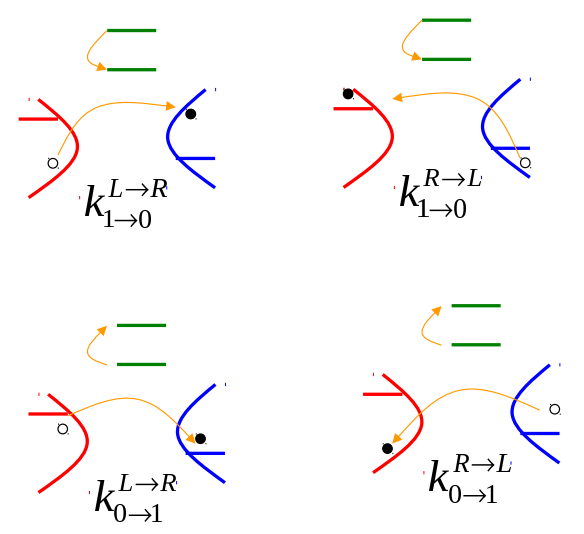

FIG. 4. Scheme of the vibrational mode excitation and relaxation processes. A full circle represents an electron transferred; a hollow circle depicts the hole that has been left behind.

A generic mechanism leading to vibrational instabilities (and eventually junction rupture) in D-A molecular rectifiers has been discussed in Ref. [26]: At large positive bias, when the D state is positioned above the acceptor level, electron-hole pair excitations by the molecular vibration (TLS) dominate the mode dynamics. This can be schematically seen in Fig. 4 . The second-order perturbation theory rate constant, to excite the vibrational mode, while transferring an electron from $L$ to $R, k_{0 \rightarrow 1}^{L \rightarrow R}$, overcomes other rates once the density of states at the left end is positioned above the density of states at the right side. This is the case at large positive bias, given our construction. The rate $k_{0 \rightarrow 1}^{L \rightarrow R}$ is defined next, in Sec. IVD,

\section{Master equation $\left(v_{d a}=0\right)$}

In the limit of weak electron-vibration coupling, once the direct tunneling term is neglected, $v_{d a}=0$, it can be shown that the population of the truncated vibrational mode satisfies a kinetic equation [27],

$$
\begin{aligned}
& \dot{p}_{1}=-\left(k_{1 \rightarrow 0}^{e}+k_{1 \rightarrow 0}^{b}\right) p_{1}+\left(k_{0 \rightarrow 1}^{e}+k_{0 \rightarrow 1}^{b}\right) p_{0}, \\
& p_{0}+p_{1}=1 .
\end{aligned}
$$

The excitation $\left(k_{0 \rightarrow 1}\right)$ and relaxation $\left(k_{1 \rightarrow 0}\right)$ rate constants are given by a Fourier transform of bath correlation functions of the operators $F_{e}$ and $F_{b}$, defined as

$$
\begin{aligned}
& F_{e}=\sum_{l, r}\left(\xi_{l, r}^{F} a_{l}^{\dagger} a_{r}+\xi_{r, l}^{F} a_{r}^{\dagger} a_{l}\right), \\
& F_{b}=\sum_{p} \xi_{p}^{B}\left(b_{p}^{\dagger}+b_{p}\right),
\end{aligned}
$$


to yield

$$
\begin{aligned}
& k_{s \rightarrow s^{\prime}}^{e}=\int_{-\infty}^{\infty} e^{i\left(\epsilon_{s}-\epsilon_{s^{\prime}}\right) \tau} \operatorname{Tr}_{F}\left[\rho_{F} F_{e}(\tau) F_{e}(0)\right] d \tau \\
& k_{s \rightarrow s^{\prime}}^{b}=\int_{-\infty}^{\infty} e^{i\left(\epsilon_{s}-\epsilon_{s^{\prime}}\right) \tau} \operatorname{Tr}_{B}\left[\rho_{B} F_{b}(\tau) F_{b}(0)\right] d \tau .
\end{aligned}
$$

Here $s=0,1$ and $\epsilon_{1}-\epsilon_{0}=\omega_{0}$. The operators are given in the interaction representation, e.g., $a_{l}^{\dagger}(t)=e^{i H_{L} t} a_{l}^{\dagger} e^{-i H_{L} t}$.

Phonon-bath induced rates. Expression (54) can be simplified, and the contribution of the phonon bath to the vibrational rates reduces to

$$
\begin{aligned}
& k_{1 \rightarrow 0}^{b}=\Gamma_{p h}\left(\omega_{0}\right)\left[f_{B}\left(\omega_{0}\right)+1\right], \\
& k_{0 \rightarrow 1}^{b}=k_{1 \rightarrow 0}^{b} e^{-\omega_{0} \beta_{p h}},
\end{aligned}
$$

where $f_{B}(\omega)=\left[e^{\beta_{p h} \omega}-1\right]^{-1}$ denotes the Bose-Einstein distribution function. The damping rate is defined as $\Gamma_{p h}(\omega)=2 J_{p h}(\omega)$,

$$
\Gamma_{p h}(\omega)=2 \pi \sum_{p}\left(\xi_{p}^{B}\right)^{2} \delta\left(\omega_{p}-\omega\right)
$$

For brevity, we ignore below the direct reference to frequency.

Electronic-baths induced rates. The electronic rate constants (54) include the following contributions [27],

$$
k_{1 \rightarrow 0}^{e}=k_{1 \rightarrow 0}^{L \rightarrow R}+k_{1 \rightarrow 0}^{R \rightarrow L} ; \quad k_{0 \rightarrow 1}^{e}=k_{0 \rightarrow 1}^{L \rightarrow R}+k_{0 \rightarrow 1}^{R \rightarrow L},
$$

satisfying

$$
\begin{aligned}
& k_{1 \rightarrow 0}^{L \rightarrow R}=2 \pi \kappa^{2} \sum_{l, r}\left|\lambda_{l}\right|^{2}\left|\lambda_{r}\right|^{2} f_{L}\left(\epsilon_{l}\right)\left(1-f_{R}\left(\epsilon_{r}\right)\right) \delta\left(\omega_{0}+\epsilon_{l}-\epsilon_{r}\right) \\
& k_{0 \rightarrow 1}^{L \rightarrow R}=2 \pi \kappa^{2} \sum_{l, r}\left|\lambda_{l}\right|^{2}\left|\lambda_{r}\right|^{2} f_{L}\left(\epsilon_{l}\right)\left(1-f_{R}\left(\epsilon_{r}\right)\right) \delta\left(-\omega_{0}+\epsilon_{l}-\epsilon_{r}\right) .
\end{aligned}
$$

Similar relations hold for the right-to-left going excitations. The energy in the Fermi function $f_{\nu}(\epsilon)$ is measured with respect to the (equilibrium) Fermi energy, placed at $\left(\mu_{L}+\mu_{R}\right)$, and we assume that the bias is applied symmetrically, $\mu_{L}=-\mu_{R}$. The rates can be expressed in terms of the fermionic $\nu=L, R$ spectral density functions

$$
J_{\nu}(\epsilon)=2 \pi \kappa \sum_{j \in \nu}\left|\lambda_{j}\right|^{2} \delta\left(\epsilon_{j}-\epsilon\right)
$$


Using Eq. (42) we resolve this as a Lorentzian function, centered around either the D or the A level,

$$
\begin{aligned}
& J_{L}(\epsilon)=\kappa \frac{\Gamma_{L}(\epsilon)}{\left(\epsilon-\epsilon_{d}\right)^{2}+\Gamma_{L}(\epsilon)^{2} / 4} \\
& J_{R}(\epsilon)=\kappa \frac{\Gamma_{R}(\epsilon)}{\left(\epsilon-\epsilon_{a}\right)^{2}+\Gamma_{R}(\epsilon)^{2} / 4} .
\end{aligned}
$$

The electronic hybridization $\Gamma_{\nu}(\epsilon)$ is given in Eq. (44). Using these definitions, we express the electronic rates [Eq. (58)] by integrals $\left(s, s^{\prime}=0,1\right)$

$$
k_{s \rightarrow s^{\prime}}^{\nu \rightarrow \nu^{\prime}}=\frac{1}{2 \pi} \int_{-\infty}^{\infty} f_{\nu}(\epsilon)\left[1-f_{\nu^{\prime}}\left(\epsilon+\left(s-s^{\prime}\right) \omega_{0}\right)\right] J_{\nu}(\epsilon) J_{\nu^{\prime}}\left(\epsilon+\left(s-s^{\prime}\right) \omega_{0}\right) d \epsilon .
$$

Observables. Within this simple kinetic approach, junction stability can be recognized by watching the TLS population in the steady-state limit: population inversion reflects on vibrational instability [27]. Solving Eq. (52) in the long time limit we find that

$$
p_{1}=\frac{k_{0 \rightarrow 1}^{e}+k_{0 \rightarrow 1}^{b}}{k_{0 \rightarrow 1}^{e}+k_{0 \rightarrow 1}^{b}+k_{1 \rightarrow 0}^{e}+k_{1 \rightarrow 0}^{b}}, \quad p_{0}=1-p_{1} .
$$

A related measure is the damping rate $K_{v i b}$ [26], depicted in Fig. 3 panel (b). It is defined as the difference between relaxation and excitation rates,

$$
K_{v i b} \equiv k_{1 \rightarrow 0}^{e}+k_{1 \rightarrow 0}^{b}-\left(k_{0 \rightarrow 1}^{e}+k_{0 \rightarrow 1}^{b}\right)
$$

Positive $K_{v i b}$ indicates on a "normal" thermal-like behavior, when relaxation processes overcome excitations. In this case, the junction remains stable in the sense that the population of the ground state is larger than the population of the excited level. A negative value for $K_{v i b}$ evinces on the process of an uncontrolled heating of the molecular mode, eventually leading to vibrational instability and junction breakdown.

In the steady-state limit, the charge current $j$, flowing from $L$ to $R$, is given by [27]

$$
j=p_{1}\left(k_{1 \rightarrow 0}^{L \rightarrow R}-k_{1 \rightarrow 0}^{R \rightarrow L}\right)+p_{0}\left(k_{0 \rightarrow 1}^{L \rightarrow R}-k_{0 \rightarrow 1}^{R \rightarrow L}\right) .
$$

This relation holds even when the TLS is coupled to an additional boson bath. Note that in the long time limit the current that is evaluated at the left end $j_{L}$ is equal to $j_{R}$. Therefore, we simple denote the current by $j$ in that limit.

Master equation calculations proceed as follows. We set the hybridization energy $\Gamma_{\nu}$ as an energy independent parameter, and evaluate the fermionic spectral functions $J_{\nu}(\epsilon)$ of Eq. (60). With this at hand, we integrate (numerically) Eq. (61), and gain the fermionic-bath 
induced rates. The phonon bath-induced rates (55) are reached by setting the parameters of the spectral function $J_{p h}$, to directly obtain $\Gamma_{p h}$, see Eq. (56). Using this set of parameters, we evaluate the levels occupation and the charge current directly in the steady-state limit. We can also time evolve the set of differential equations (52), to obtain the trajectory $p_{1,0}(t)$.

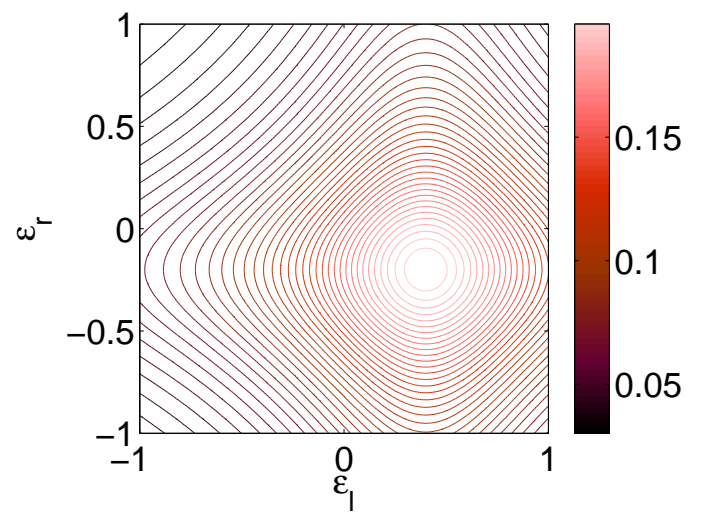

FIG. 5. Absolute value of the quantity $\pi \rho \xi_{l, r}^{F}$. The figure was generated by discretizing the reservoirs, using bands extending from $-D$ to $D, D=1$, with $N_{L}=200$ states per each band a linear dispersion relation and a constant density of states for the $H_{L, R}^{0}$ reservoirs, with a constant density of states $\rho=N_{L} / 2 D$. Electron-vibration coupling is given by $\kappa=0.1$.

\section{E. Results}

We simulate the dynamics of the subsystem in the spin-boson-fermion Hamiltonian (47) using the path-integral approach of Sec. III. In order to retrieve the vibrational mode occupation in the original basis in which Eq. (45) is written, we rotate the reduced density matrix $\rho_{S}(t)$ back to the original basis by applying the transformation $U=\frac{1}{\sqrt{2}}\left(\sigma_{x}+\sigma_{z}\right)$,

$$
\bar{\rho}_{S}(t)=U \rho_{S}(t) U
$$

The diagonal elements of $\bar{\rho}_{S}(t)$, correspond to the vibrational mode occupation, the ground state $|0\rangle$ and the excited state $|1\rangle$,

$$
p_{0}(t)=\left\langle 0\left|\bar{\rho}_{S}(t)\right| 0\right\rangle \quad p_{1}(t)=\left\langle 1\left|\bar{\rho}_{S}(t)\right| 1\right\rangle .
$$

As an initial condition we usually take $\rho_{S}(0)=\frac{1}{2}\left(-\sigma_{x}+\hat{I}_{s}\right), \hat{I}_{s}$ is a $2 \times 2$ unit matrix. Under this choice, $\bar{\rho}_{S}(0)$ has only its ground state populated. 
Our simulations are performed with the following setup, displayed in the left panel of Fig. 3. In the absence of a bias voltage we assign the donor the energy $\epsilon_{d}=-0.2$ and the acceptor the value $\epsilon_{a}=0.4$. These molecular electronic states are assumed to linearly follow the bias voltage. The right panel in Fig. 3 depicts the damping rate $K_{v i b}$ in the absence of coupling to the phonon bath, as evaluated using the Master equation method. This measure becomes negative beyond $\Delta \mu \sim 0.85$, which corresponds to the situation where the (bias shifted) donor energy exceeds the acceptor by $\omega_{0}, \epsilon_{d}-\epsilon_{a} \gtrsim \omega_{0} ; \omega_{0}=0.2$. This results in a significant exchange of electronic energy to heat, affecting junction's instability.

\section{Isolated mode}

We study the time evolution of the vibrational mode occupation using $v_{d a}=0$ (unless otherwise stated), further decoupling it from a secondary phonon bath, $K_{d}=0$.

Electron-vibration interaction energy. The interaction energy of the subsystem (TLS) to the electronic degrees of freedom is encapsulated in the matrix elements $\xi_{l, r}^{F} \equiv \kappa \lambda_{l}^{*} \lambda_{r}$, see Eq. (48). The strength of this interaction is measured by the dimensionless parameter $\pi \rho\left(\epsilon_{F}\right) \xi_{l, r}^{F}$, which connects to the phase shift experienced by Fermi sea electrons due to a scattering potential, introduced here by the vibrational mode [46]. Here, $\rho\left(\epsilon_{F}\right)$ stands for the density of states at the Fermi energy. Using the parameters of Fig. [3, taking $\kappa=0.1$, we show the absolute value of these matrix elements in Fig. 5. The contour plot is mostly limited to values smaller than 0.1, thus we conclude that this set of parameters correspond to the weak coupling limit [46]. In this limit, path-integral simulations should agree with Master equation calculations, as we indeed confirm below. Deviations should be expected at larger values, $\kappa \gtrsim 0.2$, and we study below these cases.

Units. We perform the simulations in arbitrary units with $\hbar \equiv 1$. One can scale all energies with respect to the molecule-metal hybridization $\Gamma_{\nu}$. With $\Gamma_{\nu}=1$, the weak coupling limit covers $\kappa / \Gamma_{\nu} \lesssim 0.2$. To present results in physical units, we assume that all energy parameters are given in $\mathrm{eV}$, and scale correspondingly the time unit and currents.

Dynamics. We first focus on two representative values for the bias voltage: In the lowpositive bias limit a stable operation is expected, reflected by a normal population, $p_{0}>$

$p_{1}$. At large positive bias population inversion may take place, indicating on the onset of instability and potential junction rupture [27]. 

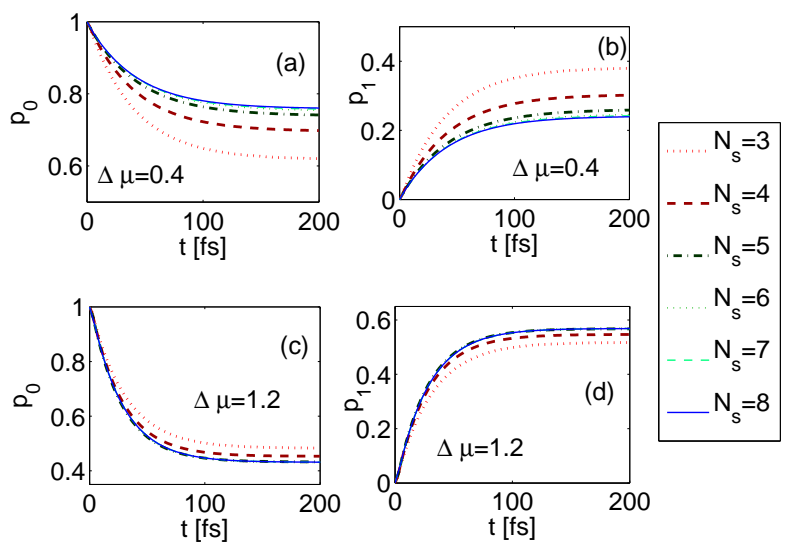

FIG. 6. Population dynamics and convergence behavior of the truncated and isolated vibrational mode (TLS) with increasing $N_{s}$. (a)-(b) Stable behavior at $\mu_{L}=-\mu_{R}=0.2$. (c)-(d) Population inversion at $\mu_{L}=-\mu_{R}=0.6$. Other parameters are the same as in Fig. 3. In all figures $\delta t=1$, $N_{s}=3$ (heavy dotted), $N_{s}=4$ (heavy dashed), $N_{s}=5$ (dashed-dotted), $N_{s}=6$ (dotted), $N_{s}=7$ (dashed) and $N_{s}=8$ (full). We used $L_{s}=30$ electronic states at each fermionic bath with sharp cutoffs at \pm 1 .

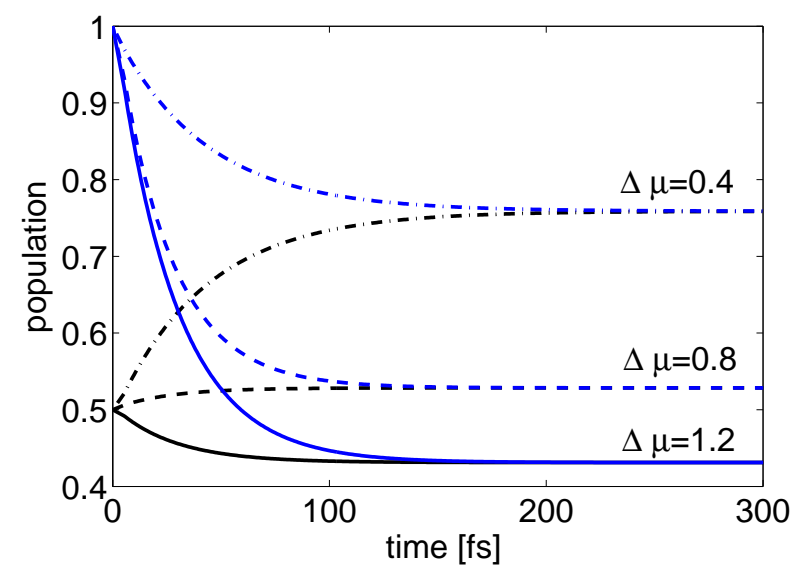

FIG. 7. Independence of the population $p_{0}$ on the initial state for different biases, $\Delta \mu=0.4,0.8$, 1.2 top to bottom. Other parameters are the same as in Fig. 3 and Fig. 6.

Fig. 6] displays the TLS dynamics, and we present data for different memory sizes $N_{s} \delta t$. At small positive bias, $\epsilon_{d}-\epsilon_{a}<\omega_{0}$, the mode occupation is "normal", $p_{0}>p_{1}$. In particular, in panels (a)-(b) we discern the case $\mu_{L}=-\mu_{R}=0.2$, resulting in the (shifted) electronic energies $\epsilon_{d}=0$ and $\epsilon_{a}=0.2$. In this case the (converged) asymptotic long-time population (representing steady-state values), are $p_{0}^{s s}=0.76$ and $p_{1}^{s s}=0.24$. In contrast, when the bias 


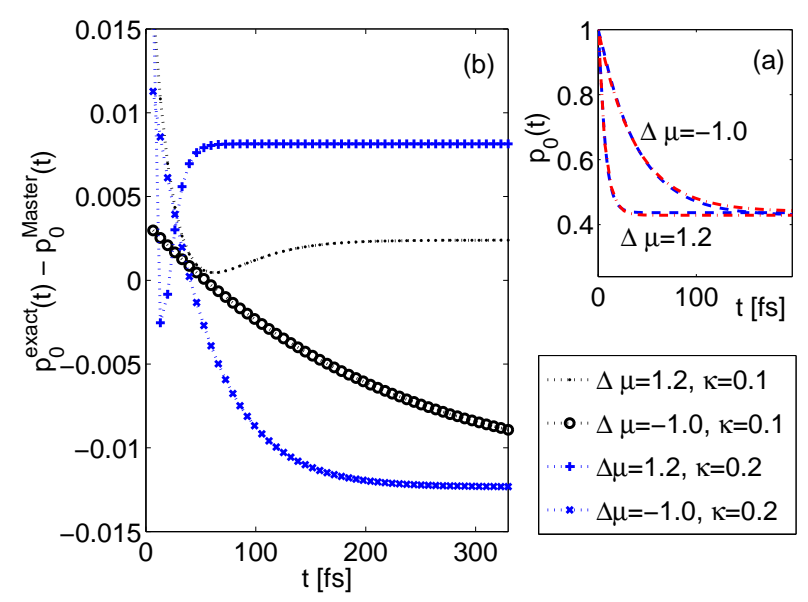

FIG. 8. Population dynamics, $p_{0}(t)$. (a) Comparison between exact simulations (dashed) and Master equation results (dashed-dotted) at $\kappa=0.2$. (b) Deviations between exact results and Master equations for $\kappa=0.1$ (dot and $\circ)$ and for $\kappa=0.2(+$ and $x)$. Other parameters are as determined in Fig. 3 ,

is large, $\mu_{L}=-\mu_{R}=0.6$, the electronic levels are shifted to $\epsilon_{d}=0.4$ and $\epsilon_{a}=-0.2$, and electrons crossing the junction discard their excess energy into the vibrational mode. Indeed, we see in Fig. 6(c)-(d) the process of population inversion, $p_{0}^{s s}=0.43$ and $p_{1}^{s s}=0.57$. The TLS approaches the steady-state value around $t_{s s} \sim 0.1$ ps. Regarding convergence behavior, we note that at large bias convergence is reached with a shorter memory size, compared to the small bias case, as expected [17].

Fig. 7 exhibits the dynamics with different initial conditions, demonstrating that the steady-state value is identical, yet the timescale to reach the stationary limit may depend on the initial state.

We compare the exact dynamics to the Master equation time evolution behavior, reached by solving Eq. (52). Panel (a) in Fig. 8 demonstrates excellent agreement for $\kappa=0.2$, for both positive and negative biases. Below we show that at this value Master equation's predictions for the charge current deviate from the exact result. Panel (b) in Fig. 8 focuses on the departure of Master equation data from the exact values. These deviations are small, but their dynamics indicate on the existence of high order excitation and relaxation rates, beyond the second order rates of Sec. IVD.

Steady-state characteristics. The full bias scan of the steady-state population is displayed in Fig. 9, and we compare path-integral results with Master equation calculations, revealing 
an excellent agreement in this weak coupling limit $(\kappa=0.1)$. The convergence behavior is presented in Fig. 10, and we plot the steady-state values as a function of memory size $\left(\tau_{c}\right)$ for three different time steps, for representative biases. The path-integral results well converge at intermediate-to-large positive biases, $\Delta \mu \gtrsim 0.2$. We had difficulty converging our results in two domains: (i) At small-positive potential bias, $\Delta \mu<0.2$. Here, large memory size should be used for reaching full convergence; decorrelation time approximately scales with $1 / \Delta \mu$. (ii) At large negative biases, $\Delta \mu<-0.4$ the current is very small as we show immediately. This implies poor convergence at the range of $\tau_{c}$ employed. At these negative biases the data oscillates with $\tau_{c}$, thus at negative bias it is the averaged value for several-large $\tau_{c}$ which is plotted in Fig. 9.

Charge current. We show the current characteristics in Fig. 11, and confirm that the junction acts as a charge rectifier. The insets display transient data, affirming that at large bias steady-state is reached faster than in the low bias case.

Strong coupling. Results at weak-to-strong couplings are shown in Fig. 12, The value of the current, as reached from Master equation calculations, scale with $\kappa^{2}$. In contrast, exact simulations indicate that the current grows more slowly with $\kappa$, and it displays clear deviations (up to 50\%) from the perturbative Master equation result at $\kappa=0.3$. Interestingly, the vibrational occupation (inset) shows little sensitivity to the coupling strength, and even at $\kappa=0.3$ the Master equation technique provides an excellent estimation for the levels occupation. This could be reasoned by the fact that excited levels occupation is given by ratio of excitation rates to the sum of excitation and relaxation rates. Such a ratio is (apparently) only weakly sensitive to the value of $\kappa$ itself, even when high-order processes do contribute to the current.

Direct tunneling vs. vibrational assisted transport. Until this point (and beyond this subsection) we have taken $v_{d a}=0$. We now evaluate the contribution of different transport mechanisms by adding a direct $\mathrm{D}-\mathrm{A}$ tunneling term, $v_{d a} \neq 0$ to our model Hamiltonian. Electrons can now either cross the junction in a coherent manner, or inelastically, by exciting/de-exciting the vibrational mode. Fig. 13 demonstrates that when the vibration assisted transport energy $\kappa$ is identical in strength to the direct tunneling element $v_{d a}$, the overall current is enhanced by about a factor of two, compared to the case when only vibrational-assisted processes are allowed. We also note that the occupation of the vibrational mode is barely affected by the opening of the new electron transmission route 


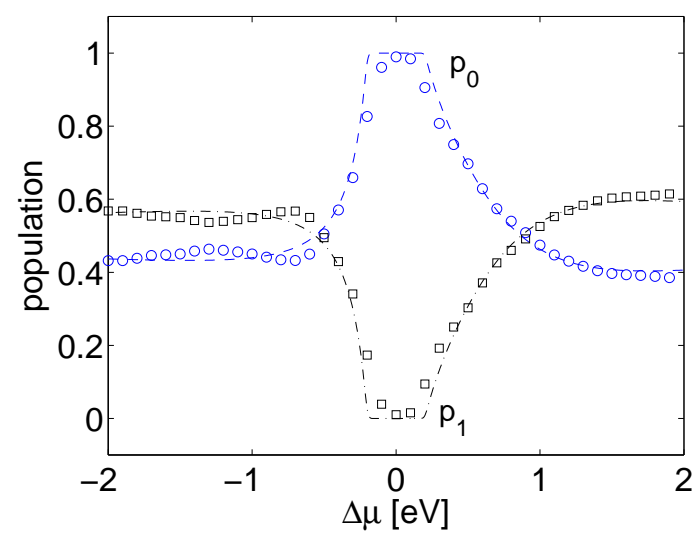

FIG. 9. Converged data for the population of the isolated vibrational mode in the steady-state limit with $\kappa=0.1$. Other parameters are the same as in Fig. 3. We display path-integral data for $p_{0}(\circ)$ and $p_{1}(\square)$. Master equation results appear as dashed line for $p_{0}$ and dashed-dotted line for $p_{1}$.

(deviations are within the convergence error). While we compare IF data to Master equation results when $v_{d a}=0$, in the general case of a nonzero D-A tunneling term perturbative methods are more involved, and techniques similar to those developed for the AH model should be used [4 18,10 - 12$]$.

\section{Equilibration with a secondary phonon bath}

We couple the isolated-truncated vibrational mode to a secondary phonon bath, and follow the mode equilibration with this bath and the removal of the vibrational instability effect, as we increase the vibrational mode-phonon bath coupling. As an initial condition, the boson bath is assumed to be thermal with an inverse temperature $\beta_{p h}$. This bath is characterized by an ohmic spectral function (50) with the dimensionless Kondo parameter $K_{d}$, characterizing subsystem-bath coupling, and the cutoff frequency $\omega_{c}$.

Population behavior. We follow the mode dynamics to the steady-state limit using the path-integral approach of Sec. III] The bosonic IF is given in the appendix. We compare exact results with Master equation predictions, and Fig. 14 depicts our simulations. The following observations can be made: (i) The vibrational instability effect is removed already for $K_{d}=0.01$, though nonequilibrium effects are still largely visible in the mode occupation. (ii) The vibrational mode is closed to be equilibrated with the phonon bath once $K_{d} \sim 0.1$. 

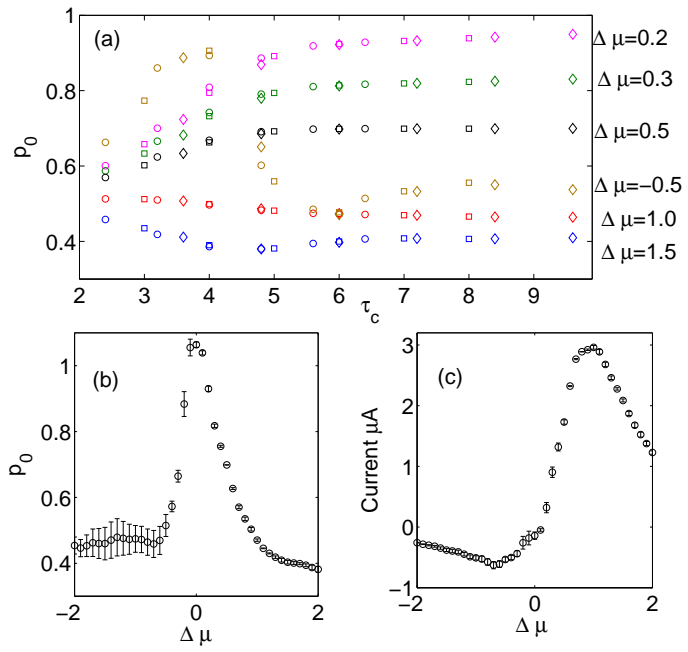

FIG. 10. (a) Convergence behavior of the population $p_{0}$ in the steady-state limit for $\kappa=0.1$. Other parameters are the same as in Fig. 3. Plotted are the steady-state values using different time steps, $\delta t=0.8(\circ), \delta t=1.0(\square)$, and $\delta t=1.2(\diamond)$ at different biases, as indicated at the right end. (b) Population mean and its standard deviation, utilizing the last six points from panel (a). (c) Current mean and its standard deviation, similarly attained from the data in panel (a).

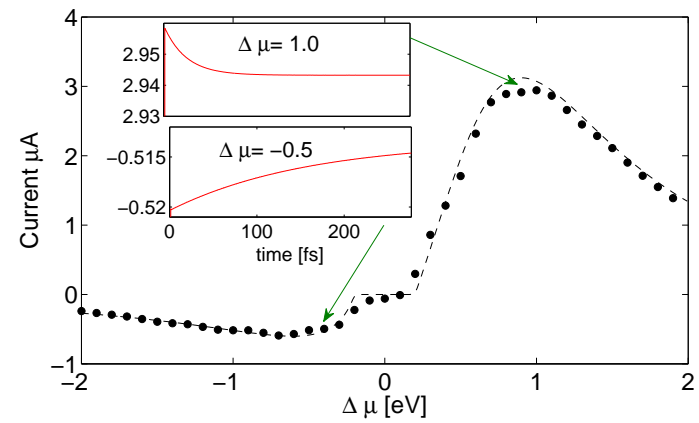

FIG. 11. Charge current in the steady-state limit for $\kappa=0.1, K_{d}=0$. Other parameters are the same as in Fig. 3. Path-integral data (o), Master equation results (dashed). The insets display transient results at $\Delta \mu=1.0 \mathrm{eV}$ (top) and $\Delta \mu=-0.5 \mathrm{eV}$ (bottom).

(iii) For the present range of parameters (large $\omega_{c}$, weak subsystem-bath couplings), Master equation tools reproduce the behavior of the vibrational mode.

Charge Current. The role of the secondary phonon bath on the charge current characteristics is displayed in Fig. 15. There are two main effects related to the presence of the phonon bath: The step structure about zero bias is flattened when $K_{d} \sim 0.1$, and the current-voltage characteristics as a whole is slightly enhanced at finite $K_{d}$, at large bias. 


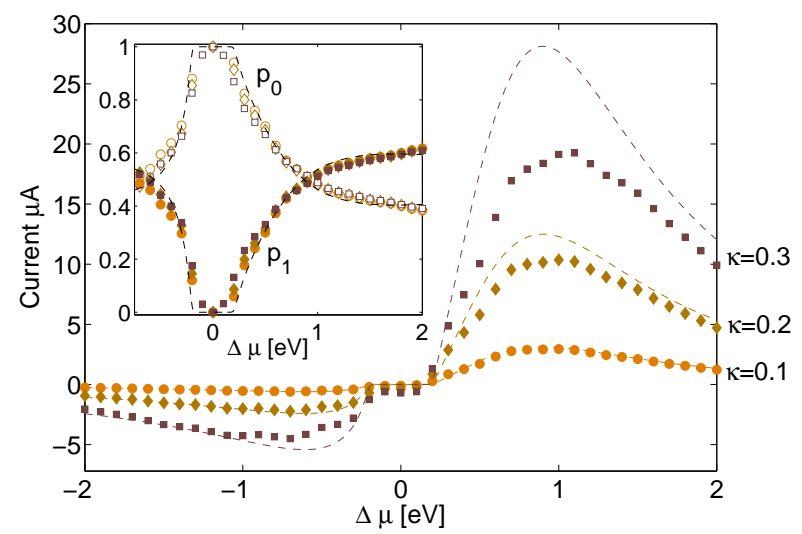

FIG. 12. Charge current and vibrational occupation in the steady-state limit at different electronvibration coupling. Path-integral data is marked by symbols, $\kappa=0.1(\circ), \kappa=0.2(\diamond)$ and $\kappa=0.3$ $(\square)$. The corresponding Master equation results appear as dashed lines. Inset: The population behavior in the steady-state limit for the three cases $\kappa=0.1(\circ), \kappa=0.2(\diamond)$ and $\kappa=0.3(\square)$, with empty symbols for $p_{0}$ and filled ones for $p_{1}$. Other parameters are the same as in Fig. 3.

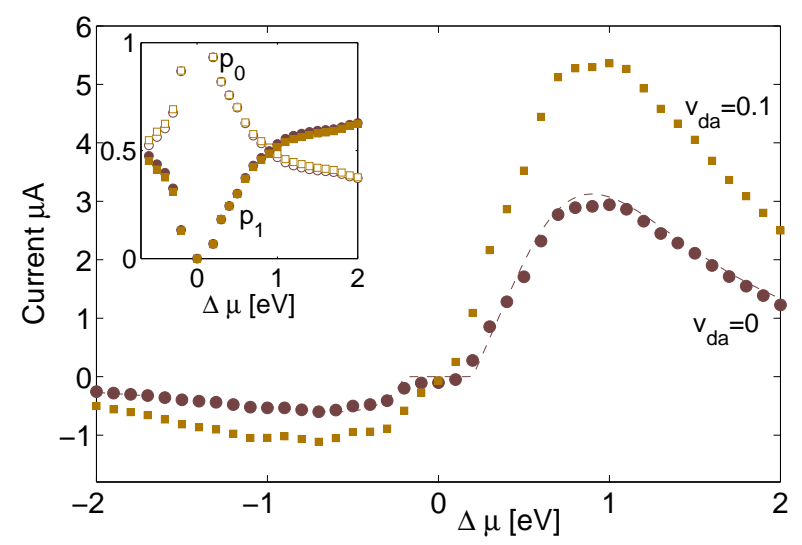

FIG. 13. Study of the contribution of different transport mechanisms. $v_{d a}=0$ (०), with Master equation results noted by the dashed line, and $v_{d a}=0.1(\square)$. The main plot displays the charge current. The inset presents the vibrational levels occupation, with empty symbols for $p_{0}$ and filled symbols for $p_{1}$. Other parameters are the same as in Fig. 3, particularly, the vibrational-electronic coupling is $\kappa=0.1$.

Both of these effects are excellently reproduced with the Master equation, and we conclude that in this weak-coupling regime the presence of the phonon bath does not affect the rectifying behavior of the junction. We have also verified (not shown) that at stronger coupling, $\kappa=0.2$ (where Master equation fails), the thermal bath similarly affects the current-voltage 
behavior.

An important observation is that the current itself does not testify on the state of the vibrational mode, whether it is in a stable or an unstable nonequilibrium state, and whether it is thermalized. The study of the current characteristics itself ( $j$ vs. $\Delta \mu$ ) is therefore insufficient to determine junction stability. More detailed information can be gained from the structure of the first derivative, $d j / d(\Delta \mu)$, the local density of states, and the second derivative, $d^{2} j / d \Delta \mu^{2}$, providing spectral features [47-49]. In order to examine these quantities, our simulations should be performed with many more bath states, to eliminate possible spurious oscillations in the current (of small amplitudes) that may result from the finite discretization of the fermi baths.

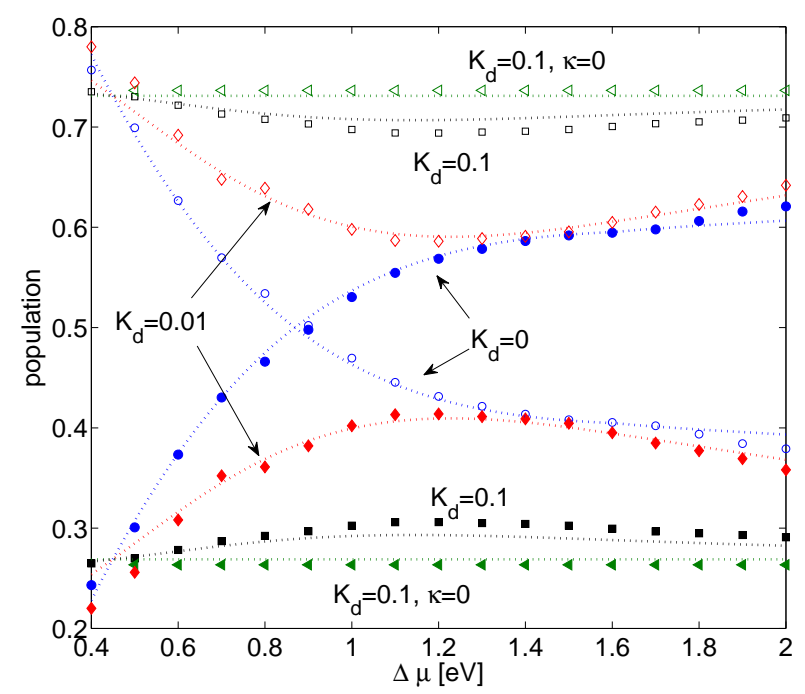

FIG. 14. Equilibration of the molecular vibrational mode with increasing coupling to a secondary phonon bath. Path-integral results, (full symbols for $p_{1}$, and empty symbols for $p_{0}$ ) with $K_{d}=0$ $(\circ), K_{d}=0.01(\diamond), K_{d}=0.1(\square)$, and, $K_{d}=0.1, \kappa=0(\triangleleft)$. Unless otherwise specified, $\kappa=0.1$, $\beta_{p h}=5$ and the spectral function follows (50) with $\omega_{c}=15$. All other electronic parameters are the same as in Fig. 3, Master equation results appear in dotted lines.

\section{F. Convergence and Computational aspects}

Convergence of the path-integral method should be verified with respect to three numerical parameters: the number of states used to mimic a fermi sea, $L_{s}$, the time step adopted, 


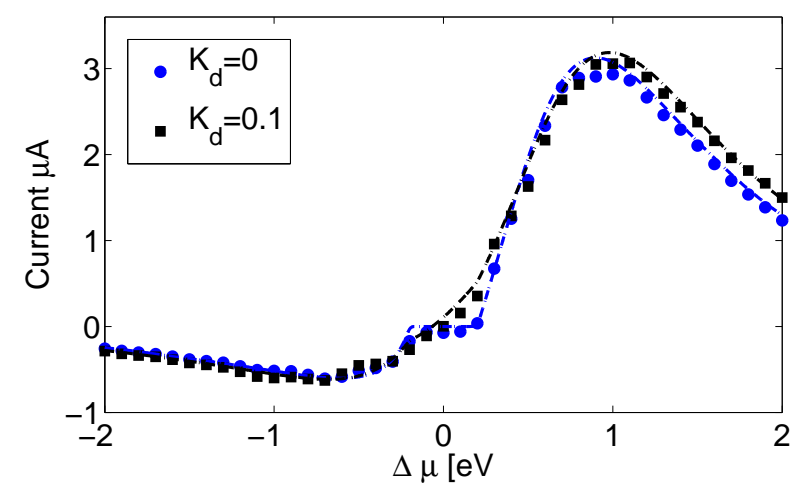

FIG. 15. Charge current for an isolated mode, $K_{d}=0$ (०), and an equilibrated mode, $K_{d}=0.1$, $\beta_{p h}=5, \omega_{c}=15(\square)$. Other electronic parameters are given in Fig. 3. Master equation results appear in dashed-dotted lines.

$\delta t$, and the memory time accounted for, $\tau_{c}$. (i) Fermi sea discretization. We have found that excellent convergence is achieved for relatively "small" fermi reservoirs, taking into account $L_{s}>20$ states for each reservoir. In our simulations we practically adopted $L_{s}=30$ for each Fermi bath. (ii) Time-step discretization. The first criteria in selecting the value of the time step $\delta t$ is that dynamical features of the isolated vibrational mode should be observed. Using $\omega_{0}=0.2$, the period of the bath-free Rabbi oscillation is $2 \pi /\left(\omega_{0}\right) \sim 30$, thus a time step of $\delta t \sim 1$ can capture the details of the TLS oscillation. This consideration serves as an "upper bound" criteria. The second consideration connects to the time discretization error, originates from the approximate splitting of the total time evolution operator into a product of terms, see Eq. (5). For the particular Trotter decomposition employed, the leading error grows with $\delta t^{3} \times\left(\left[H_{S},\left[V, H_{S}\right]\right] / 12+\left[V,\left[V, H_{S}\right]\right] / 24\right)[50]$ where $V=V_{S B}+V_{S F}+H_{B}+H_{F}$. The decomposition is exact when the coupling of the subsystem to the reservoirs is weak and the time-step is small, $\delta t \rightarrow 0$. For large coupling one should take a sufficiently small time-step in order to avoid significant error buildup. In the preset work, the dimensionless coupling to the fermi sea $\pi \rho \kappa \lambda_{l}^{*} \lambda_{r}$ is typically maintained lower that 0.3 ; the dimensionless coupling to the boson bath is taken as $K_{d}=0.1$. The value of $\delta t=0.6-1.2$ is thus sufficiently small for our simulations. (iii) Memory error. Our approach assumes that bath correlations exponentially decay resulting from the finite temperature and the nonequilibrium condition. Based on this assumption, the total influence functional was truncated to include only a finite number of time steps $N_{s}$, where $\tau_{c}=N_{s} \delta t$. The total IF is retrieved 
by taking the limit $N_{s} \rightarrow N,(N=t / \delta t)$. Our simulations were performed for $N_{s}=3 \ldots 9$, covering memory time up to $\tau_{c}=N_{s} \delta t \sim 10$. The results displayed converged for $N_{s} \sim 7-9$ for $\delta t=1$.

Computational efforts can be partitioned into two parts: In the initialization step the (time invariant) IFs are computed. The size of the fermionic IF is $d^{2 N_{s}}$, where $d$ is the dimensionality of the subsystem (two in our simulations). The power of two at the exponent results from the forward and backward time evolution operators in the path-integral expression. This initialization effort thus scales exponentially with the memory size accounted for. The preparation of the bosonic IF is more efficient if the FV IF is used [16]. In the second, time evolution, stage, we iteratively apply the linear map (27) or (34), a multiplication of two objects of length $d^{2 N_{s}}$. This operation linearly scales in time.

We now comment on the simulation time of a convergence analysis as presented in Fig. 10, covering three different time steps and $N_{s}=3, \ldots, 9$. The MATLAB implementation of the computational algorithm took advantage of the MATLAB built-in multi-threaded parallel features and utilized $100 \%$ of all available CPU cores on a node. When executed on one cluster node with two quad-core 2.2GHz AMD Opteron cpus and 16GB memory, convergence analysis for of the full voltage scan took about 4x24 hours and 250MB of memory. Computations performed on the GPC supercomputer at the SciNet HPC Consortium [51] were three times faster. Computational time scales linearly with the simulated time $t$. For a fixed $N_{s}$ value, the computational effort does not depend on the system temperature and other parameters employed.

\section{SUMMARY}

We have developed an iterative numerically-exact path-integral scheme that can follow the dynamics, to the steady-state limit, of subsystems coupled to multiple bosonic and fermionic reservoirs in an out-of-equilibrium initial state. The method is based on the truncation of time correlations in the influence functional, beyond the memory time dictated by temperature and chemical biases. It combines two techniques: the QUAPI method [16], for treating the dynamics of subsystems coupled to harmonic baths, and the INFPI approach [17], useful for following the evolution of a subsystem when interacting with fermionic baths.

The method is stable, efficient, and flexible, and it allows one to achieve transient and 
steady-state data for both the reduced density matrix of the subsystem and expectation values of operators, such as the charge current and energy current. The method can be viewed as an extension of QUAPI, to incorporate fermions in the dynamics. It could be further expanded to include time-dependent Hamiltonians, e.g., pulsed fields.

To demonstrate the method usability in the field of molecular conduction, we have applied the general scheme, and studied vibrational dynamics in a molecular rectifier setup, where vibrational equilibration with an additional phonon bath is allowed. Our main conclusions in this study are the following: (i) The vibrational instability effect disappears once the vibrational mode is weakly coupled $\left(K_{d} \sim 0.01\right)$ to an additional phonon bath that can dissipate the excess energy. (ii) When $K_{d} \sim 0.1$, the vibrational mode is equilibrated with the secondary phonon bath. (iii) The charge current does not testify on vibrational heating and instability. While we have performed those simulations using a truncated vibrational mode, a TLS, representing an anharmonic mode, we argue that the main characteristics of the vibrational instability effect remain intact when the selected mode is made harmonic [27].

Our simulations indicate that Master equation methods can excellently reproduce exact results at weak coupling, in the markovian limit. More significantly, Master equation tools can be used beyond the weak coupling limit $(\kappa \sim 0.3)$, if only a qualitative understanding of the junction behavior is inquired. One should note that our Master equation technique treats the D and A coupling to the metals exactly. It is perturbative only in the interaction of the vibrational mode to the electrons, and to other phonon degrees of freedom. In the case where tunneling transmission competes with phonon-assisted transport, only pathintegral simulations were provided, as more involved Master equation methodologies should be developed in this case.

Our future objectives are twofold: (i) to improve the time-evolution algorithm, and (ii) to employ the method for the study of other problems in molecular electronics and phononics. By improving the methodology, we would like to extend the usability of our method to difficult parameter regimes (strong coupling), e.g., by developing an equation-of-motion for the memory function [52, 53]. This will also allow us to simulate more feasibly the dynamics of an $n$-level subsystem. Another related objective is the study of heat current characteristics in the spin-boson molecular junction [54]. The single-bath spin-boson model displays a rich dynamics with a complex phase diagram. Similarly, we expect that the nonequilibrium 
version, with two harmonic baths of different temperatures coupled to the TLS, will show complex behavior for its heat current- temperature characteristics. Recent results, obtained using an extension of the noninteracting blip approximation to the nonequilibrium regime [55], demonstrate rich behavior. Other problems that could be directed with our method include plexcitonics systems, as the coupling between surface plasmons and molecular excitons should be treated beyond the perturbative regime [56]. Finally, we have discussed the calculation of reduced density matrix and currents in the path-integral framework. It is of interest to generalize these expressions and gain higher order cumulants, for the study of current, noise, and fluctuation relations in many-body out-of-equilibrium systems.

\section{ACKNOWLEDGMENTS}

DS acknowledges support from an NSERC discovery grant. The work of LS was supported by an Early Research Award of DS. Computations were performed on the GPC supercomputer at the SciNet HPC Consortium [51]. SciNet is funded by: the Canada Foundation for Innovation under the auspices of Compute Canada; the Government of Ontario; Ontario Research Fund - Research Excellence; and the University of Toronto.

APPENDIX A: TIME-DISCRETE FEYNMAN-VERNON INFLUENCE FUNCTIONAL

With the discretization of the path, the influence functional takes the form (17). The coefficients $\eta_{k, k^{\prime}}$ were given in [16] and we include them here for the completeness of our presentation. The expressions are given here for the case of a single boson bath with the

initial temperature $1 / \beta_{p h}$ and the spectral function $J_{p h}(\omega)=\pi \sum_{p}\left(\xi_{p}^{B}\right)^{2} \delta\left(\omega-\omega_{p}\right), J_{p h}(\omega)=$ $J_{p h}(-\omega)$, 


$$
\begin{aligned}
\eta_{k, k^{\prime}} & =\frac{2}{\pi} \int_{-\infty}^{\infty} d \omega \frac{J_{p h}(\omega)}{\omega^{2}} \frac{\exp \left(\beta_{p h} \omega / 2\right)}{\sinh \left(\beta_{p h} \omega / 2\right)} \sin ^{2}(\omega \delta t / 2) e^{-i \omega \delta t\left(k-k^{\prime}\right)}, \quad 0<k^{\prime}<k<N \\
\eta_{k, k} & =\frac{1}{2 \pi} \int_{-\infty}^{\infty} d \omega \frac{J_{p h}(\omega)}{\omega^{2}} \frac{\exp \left(\beta_{p h} \omega / 2\right)}{\sinh \left(\beta_{p h} \omega / 2\right)}\left(1-e^{-i \omega \delta t}\right), \quad 0<k<N \\
\eta_{k, 0} & =\frac{2}{\pi} \int_{-\infty}^{\infty} d \omega \frac{J_{p h}(\omega)}{\omega^{2}} \frac{\exp \left(\beta_{p h} \omega / 2\right)}{\sinh \left(\beta_{p h} \omega / 2\right)} \sin (\omega \delta t / 4) \sin (\omega \delta t / 2) e^{-i \omega(k \delta t-\delta t / 4)}, \quad 0<k<N \\
\eta_{N, k^{\prime}} & =\frac{2}{\pi} \int_{-\infty}^{\infty} d \omega \frac{J_{p h}(\omega)}{\omega^{2}} \frac{\exp \left(\beta_{p h} \omega / 2\right)}{\sinh \left(\beta_{p h} \omega / 2\right)} \sin (\omega \delta t / 4) \sin (\omega \delta t / 2) e^{-i \omega\left(N \delta t-k^{\prime} \delta t-\delta t / 4\right)}, \quad 0<k^{\prime}<N \\
\eta_{N, 0} & =\frac{2}{\pi} \int_{-\infty}^{\infty} d \omega \frac{J_{p h}(\omega)}{\omega^{2}} \frac{\exp \left(\beta_{p h} \omega / 2\right)}{\sinh \left(\beta_{p h} \omega / 2\right)} \sin ^{2}(\omega \delta t / 4) e^{-i \omega(N \delta t-\delta t / 2)} \quad \text { (A1) } \\
\eta_{0,0}= & \eta_{N, N}=\frac{1}{2 \pi} \int_{-\infty}^{\infty} d \omega \frac{J_{p h}(\omega)}{\omega^{2}} \frac{\exp \left(\beta_{p h} \omega / 2\right)}{\sinh \left(\beta_{p h} \omega / 2\right)}\left(1-e^{-i \omega \delta t / 2}\right)
\end{aligned}
$$

[1] J. Bonca and S. Trugmann, Phys. Rev. Lett. 75, 2566 (1995).

[2] H. Ness, S. Shevlin, and A. Fisher, Phys. Rev. B 63, 125422 (2001).

[3] M. Czek, M. Thoss, and W. Domcke, Phys. Rev. B 70, 125406 (2004).

[4] M. Galperin, M. A. Ratner, and A. Nitzan, J. Phys.: Condens. Matter 19, 103201 (2007).

[5] A. Mitra, I. Aleiner, and A. J. Millis, Phys. Rev. B 69, 245302 (2004).

[6] M. Galperin, A. Nitzan, and M. A. Ratner, Phys. Rev. B 75, 155312 (2007).

[7] M. Galperin, M. A. Ratner, and A. Nitzan, J. Chem. Phys 130, 144109 (2009).

[8] J. Fransson and M. Galperin, Phys. Rev. B 81, 075311 (2010).

[9] D. Segal and A. Nitzan, J. Chem. Phys. 117, 3915 (2002).

[10] M. Leijnse and M. R. Wegewijs, Phys. Rev. B 78, 235424 (2008).

[11] R. Hartle and M. Thoss, Phys. Rev. B 83, 125419 (2011).

[12] R. Hartle and M. Thoss, Phys. Rev. B 83, 115414 (2011).

[13] R. Volkovich, R. Hartle, and M. Thoss, Phys. Chem. Chem. Phys. 32, 14333 (2010).

[14] D. W. H. Swenson, T. Levy, G. Cohen, E. Rabani, and W. H. Miller, J. Chem. Phys. 134, 164103 (2011).

[15] D. W. H. Swenson, G. Cohen, and E. Rabani, Molecular Physics 110, 743 (2012).

[16] N. Makri and D. E. Makarov, J. Chem. Phys. 102, 4600 (1995); 102, 4611 (1995).

[17] D. Segal, A. J. Millis, and D. R. Reichman, Phys. Rev. B 82, 205323 (2010); Phys. Chem. Chem. Phys. 13, 14378 (2011). 
[18] S. Bedkihal and D. Segal, Phys. Rev. B 85, 155324 (2012).

[19] M. Kulkarni, K. L. Tiwari, and D. Segal, Phys. Rev. B 86, 155424 (2012); New J. Phys. 15, $013014(2013)$.

[20] L. Muhlbacher and E. Rabani, Phys. Rev. Lett. 100, 176403 (2008).

[21] H. Wang, I. Pshenichnyuk, R. Hartle, and M. Thoss, J. Chem. Phys. 135, 244506 (2011).

[22] S. Weiss, J. Eckel, M. Thorwart, and R. Egger, Phys. Rev. B 77, 195316 (2008).

[23] J. Eckel, F. Heidrich-Meisner, S. G. Jakobs, M. Thorwart, M. Pletyukhov, and R. Egger, New J. Phys. 12, 043042 (2010).

[24] R. Hutzen, S. Weiss, M. Thorwart, and R. Egger, Phys. Rev. B 85, 121408 (2012).

[25] A. Aviram and M. A. Ratner, Chem. Phys. Lett. 29, 277 (1974).

[26] J.-T. Lu, P. Hedegard, and M. Brandbyge, Phys. Rev. Lett. 107, 046801 (2011).

[27] L. Simine and D. Segal, Phys. Chem. Chem. Phys. 14, 13820 (2012).

[28] J. Kondo, Prog. Theor. Phys. 32, 37 (1964).

[29] A. Mitra and A. J. Millis, Phys. Rev. B 72, 121102(R) (2005).

[30] A. Mitra and A. J. Millis, Phys. Rev. B 76, 085342 (2007).

[31] D. Segal, D. R. Reichman, and A. J. Millis, Phys. Rev. B 76, 195316 (2007).

[32] R. M. Lutchyn, L. Cywinski, C. P. Nave, and S. D. Sarma, Phys. Rev. B 78, 024508 (2008).

[33] R. P. Feynman and A. R. Hibbs, Quantum Mechanics and Path Integrals (McGraw-Hill, NewYork, 1965).

[34] I. Klich, "in quantum noise in mesoscopic systems," (Kluwer, 2003).

[35] L. H. Yu., Z. K. Keane, J. W. Ciszek, L. Cheng, M. P. Stewart, J. M. Tour, and D. Natelson, Phys. Rev. Lett. 93, 266802 (2004).

[36] D. Djukic, K. S. Thygesen, C. Untiedt, R. H. M. Smit, K. Jacobsen, and J. M. van Ruitenbeek, Phys. Rev. B 71, 161402 (2005).

[37] M. Kumar, R. Avriller, A. L. Yeyati, and J. M. van Ruitenbeek, Phys. Rev. Lett. 108, 146602 (2012).

[38] A. N. Pasupathy, J. Park, C. Chang, A. V. Soldatov, R. C. B. S. Lebedkin, J. E. Grose, L. A. K. Donev, J. P. Sethna, D. C. Ralph, and P. L. McEuen, Nano Lett. 5, 203 (2005).

[39] Z. Ioffe, T. Shamai, A. Ophir, G. Noy, I. Yutsis, K. Kfir, O. Cheshnovsky, and Y. Selzer, Nature Nanotech. 3, 727 (2008).

[40] D. R. Ward, D. A. Corley, J. M. Tour, and D. Natelson, Nature Nanotech. 6, 33 (2011). 
[41] Z. Huang, F. Chen, R. Dagosta, P. A. Bennett, M. D. Ventra, and N. Tao, Nature Nanotech. 2, 698 (2007).

[42] O. Entin-Wohlman, Y. Imry, and A. Aharony, Phys. Rev. B 82, 115314 (2010).

[43] J.-H. Jiang, O. Entin-Wohlman, and Y. Imry, Phys. Rev. B 85, 075412 (2012).

[44] O. Entin-Wohlman and A. Aharony, Phys. Rev. B 85, 085401 (2012).

[45] G. D. Mahan, Many-particle physics (Plenum press, New York, 2000).

[46] B. Roulet, J. Gavoret, and P. Nozieres, Phys. Rev. 178, 1072 (1969).

[47] T. Mii, S. Tikhodeev, and H. Ueba, Phys. Rev. B 68, 205406 (2003).

[48] M. Galperin, M. Ratner, and A. Nitzan, J. Chem. Phys. 121, 11965 (2004).

[49] M. Galperin, M. Ratner, and A. Nitzan, Nano. Lett. 4, 1605 (2004).

[50] D. J. Tannor, Intriduction to Quantum Mechanics: A time dependent perspective (University Science Books, 2007).

[51] C. Loken and et al., J. Phys.: Conf. Ser. 256, 012026 (2010).

[52] A. A. Golosov, R. A. Friesner, and P. Pechukas, J. Chem. Phys 110, 138 (1999).

[53] G. Cohen and E. Rabani, Phys. Rev. B 84, 075150 (2011).

[54] D. Segal and A. Nitzan, Phys. Rev. Lett. 94, 034301 (2005); J. Chem. Phys. 122, 194704 (2005).

[55] L. Nicolin and D. Segal, J. Chem. Phys. 135, 164106 (2011).

[56] A. Manjavacas, F. J. G. de Abajo, and P. Nordlander, Nano Lett. 11, 2318 (2011). 